\title{
MULTIPLICITY RESULTS FOR PERIODIC SOLUTIONS OF SECOND ORDER ODES WITH ASYMMETRIC NONLINEARITIES
}

\author{
C. REBELO AND F. ZANOLIN
}

\begin{abstract}
We prove various results on the existence and multiplicity of harmonic and subharmonic solutions to the second order nonautonomous equation $x^{\prime \prime}+g(x)=s+w(t, x)$, as $s \rightarrow+\infty$ or $s \rightarrow-\infty$, where $g$ is a smooth function defined on a open interval $] a, b[\subset \mathbb{R}$. The hypotheses we assume on the nonlinearity $g(x)$ allow us to cover the case $b=+\infty$ (or $a=-\infty)$ and $g$ having superlinear growth at infinity, as well as the case $b<+\infty$ (or $a>-\infty$ ) and $g$ having a singularity in $b$ (respectively in $a$ ). Applications are given also to situations like $g^{\prime}(-\infty) \neq g^{\prime}(+\infty)$ (including the so-called "jumping nonlinearities"). Our results are connected to the periodic Ambrosetti - Prodi problem and related problems arising from the Lazer - McKenna suspension bridges model.
\end{abstract}

\section{INTRODUCTION}

In this paper we deal with the existence and multiplicity of periodic solutions (harmonics and subharmonics) for a nonautonomous second order scalar differential equation depending on a real parameter $s$. More precisely, following [15] and [27], we deal with an equation of the form

$$
x^{\prime \prime}+g(x)=s+w(t, x), \quad s \in \mathbb{R},
$$

where $g: J \rightarrow \mathbb{R}$ and $w: \mathbb{R} \times J \rightarrow \mathbb{R}$ are continuous functions and $J \subset \mathbb{R}$ is an open interval. Throughout the article we assume that $w(\cdot, x)$ is periodic of a fixed period $T>0$, for any $x \in J$ and $w$ is also globally bounded on its domain.

In what follows, we present some results concerning the way in which a modification of the parameter $s$ affects the number of the periodic solutions for equation $(E)_{s}$. This scope will be achieved by assuming on $g$ some conditions that, in the case of $J=\mathbb{R}$, imply a different behaviour at $\pm \infty$ for $g(x) / x$ or $g^{\prime}(x)$ (the case of so-called "jumping or asymmetric nonlinearities"). Problems of this form are

Received by the editors August 4, 1994 and, in revised form, February 28, 1995.

1991 Mathematics Subject Classification. Primary 34C25; Secondary 34B15.

Key words and phrases. Periodic solutions, subharmonics, asymmetric nonlinearities, PoincaréBirkhoff fixed point theorem.

Work performed in the frame of the EEC project "Non linear boundary value problems: existence, multiplicity and stability of solutions", grant ERB CHRX-CT94-0555.

The first author is on leave of absence from Faculdade de Ciências da Universidade de Lisboa with a fellowship from Programa Ciência (JNICT).

The second author's work performed under the auspices of GNAFA-CNR and supported by MURST (40\% and $60 \%$ funds). 
usually known in the literature as "of Ambrosetti - Prodi type", due to the seminal paper [1]. Indeed, in [1], and made more precise in [2], for $J=\mathbb{R}$ and $g$ of class $\mathcal{C}^{2}$ such that $g^{\prime \prime}>0$ and $g^{\prime}(-\infty)<\lambda_{1}<g^{\prime}(+\infty)<\lambda_{2}$, it was proved that for the equation

$$
x^{\prime \prime}+g(x)=\operatorname{sh}(t)
$$

with $h(t)=\sin ((\pi / T) t)$ (or for a more general elliptic PDE), there is $s_{0}$ such that $(D)_{s}$ has no solutions, one solution, or two solutions satisfying the Dirichlet boundary condition $x(0)=x(T)=0$, when $s<s_{0}, s=s_{0}$, or $s>s_{0}$, respectively. Note that $h(t)$ in $(D)_{s}$ is the eigenfunction corresponding to the first eigenvalue $\lambda_{1}=(\pi / T)^{2}$ for the Dirichlet problem on the interval $[0, T]$.

Since the appearance of [1], a wide number of existence and multiplicity results were obtained in the same direction and much attention has been devoted to the case when the interval $] g^{\prime}(-\infty), g^{\prime}(+\infty)$ [ contains one or more higher order eigenvalues. Without mentioning various interesting theorems on the Dirichlet problem for elliptic equations, if we confine ourselves to the case of the two-point BVP for scalar ODEs, we can find in the literature sharp estimates concerning the number of the solutions (see [23], [40], [29], [3], [27], [5], [30], [43] and the references therein). In particular, we recall a recent article by Zinner [43] where, for $g$ of class $\mathcal{C}^{1}$ on $J=\mathbb{R}$, and $g^{\prime}(-\infty)=\alpha \in \mathbb{R}, g^{\prime}(+\infty)=+\infty$, it is proved that for any $n \in \mathbb{Z}_{0}^{+}$(the set of positive integers), there exists $s_{n}$ such that $(D)_{s}$ has at least $2 n$ solutions, satisfying the two-point boundary condition, for each $s>s_{n}$.

In [15], Fabry, Mawhin and Nkashama initiated the study of the Ambrosetti - Prodi problem with periodic boundary conditions. In this situation, the eigenfunction corresponding to the first eigenvalue $\lambda_{0}=0$ is $h(t) \equiv 1$. Then, the general second order equation to analyse takes the form $x^{\prime \prime}+f\left(t, x, x^{\prime}\right)=s$ (see [15, p. 174]). In [36], Ortega discussed the Ambrosetti - Prodi periodic problem for a damped Duffing equation, from the point of view of the stability of the solutions. In the present article we consider a situation which is complementary with respect to [36] and therefore here and subsequently, we don't consider possible dependence on $x^{\prime}$ and confine ourselves to the investigation of the conservative case $x^{\prime \prime}+f(t, x)=s$. This justifies our choice of $(E)_{s}$ for the periodic problem. Another motivation to study $(E)_{s}$ with $g$ a jumping nonlinearity comes from the interest in the analysis of the harmonic and subharmonic solutions for the Lazer - McKenna model of oscillations in suspension bridges. Indeed, we notice that in [27, p. 553], Lazer and McKenna propose the study of the periodic solutions of an equation of the form $x^{\prime \prime}+g(x)=s+w(t)$, with $g^{\prime}(-\infty) \neq g^{\prime}(+\infty)$. For sake of completeness, we should mention that variants of $(E)_{s}$ were considered in [25] and [8], having at the right hand side of the equation a term of the form $s(1+q(t))$, with $|q|_{\infty}<<1$. However, in order to unify the presentation of our results, we discuss only the periodic problem for $(E)_{s}$, leaving to the interested reader the care of completing the missing details for possible variants. With this presentation, we can now recall a theorem of del Pino, Manásevich and Murua in [8] where it is assumed, for $g$ of class $\mathcal{C}^{1}$ on $J=\mathbb{R}$, the existence of positive integers $k$ and $n$ such that $\lambda_{k-1}<\alpha=g^{\prime}(-\infty)<\lambda_{k} \leq \lambda_{n}<\beta=g^{\prime}(+\infty)<\lambda_{n+1}$, with $\lambda_{j}=(2 \pi / T)^{2} j^{2}$ the $j$-th positive eigenvalue for the $T$-periodic problem, and the existence of at least $2(n-l)+1$ solutions of period $T$ to $(E)_{s}$, for sufficiently large positive $s$, is proved. Here $l$ denotes the integer part of $(T / \pi)(\sqrt{\alpha \beta} /(\sqrt{\alpha}+\sqrt{\beta}))$. 
After this introductory presentation, we pass to the description of our main theorems. The aim of our work is threefold:

- we extend Zinner's result to the periodic problem and, at the same time, unify it with the above recalled theorem of del Pino, Manásevich and Murua, allowing the possibility that $g^{\prime}$ be bounded or unbounded and also that it has neither limits at $\pm \infty$, nor the same bounds as in [8];

- we provide a unique proof which is valid both to the case of bounded and unbounded intervals. Thus, in particular, we are able to deal with the standard case $J=\mathbb{R}$ previously considered by all the authors, as well as with equations with singularities. To this end, we take advantage from an observation derived in [18] where it is shown that, under very natural conditions, the effects of a singular nonlinearity are the same like that of a superlinear term for the periodic problem;

- we produce a unique setting for proving the existence of harmonic solutions (i.e. $T$-periodic solutions) and, for any fixed integer $m$, subharmonics of order $m$ (i.e. periodic solutions of minimal period $m T$ in the class of the periods $j T$ with $1 \leq j \leq m)$.

The argument of the proof is developed through some steps. First of all we write equation $(E)_{s}$ as an equivalent planar system and apply the Poincaré - Birkhoff fixed point theorem to the associated Poincaré's map or its iterates. In order to verify the "twist condition" (which is the crucial assumption for the application of this theorem), we have to estimate the number of rotations of the trajectories around some fixed point in the plane. At that moment we need to introduce suitable polar coordinates for the evaluation of the angular gaps. Usually this is performed by means of the classical Prüfer transformation. For our purposes, however, it would be convenient to use at the same time different scales for small and large trajectories, in order to take into account either the possibility of singularities or of superlinear growths. Indeed, in these cases, a nonlinear change of variable introduced by Conti in [4] turns out to be particularly useful in simplifying some technical estimates in the proof, but, on the other hand, such a choice of "deformed" polar coordinates, needs to be accompanied by a precise evaluation of the number of rotation in terms of the standard polar coordinates. Thus, in Section 2, we describe a general setting for a large class of change of variables and introduce some constants which are useful for translating the information obtained within one polar coordinates system to another one. In Section 3 we give some general results for the global continuability of the solutions to the Cauchy problems associated to $(E)_{s}$, in order to have the Poincaré operator well defined. In this context we also provide a suitable truncation showing that we can always reduce our domain to the cases $J=(-\infty, b[$ (and the symmetric one $J=] a,+\infty)$ ) or $J=\mathbb{R}$. After the presentation of all these preliminary technical tools, in Section 4 we are in position to prove the main results. Precisely, we pass to the planar system

$$
x^{\prime}=y, \quad y^{\prime}=-(g(x)-s)+w(t, x)
$$

and using some time mapping estimates we evaluate the rotation number of the trajectories around the point $P_{s}:=\left(g^{-1}(s), 0\right)$, where the meaning of $g^{-1}(s)$ will be clear from the context. Then we can prove that solutions starting near the point $P_{s}$ rotate faster than large norm solutions and thus the twist condition is verified. We notice that for our proof we need the uniqueness of the solutions to the Cauchy problems associated to $(E)_{s}$. This will be guaranteed by smoothness or 
local lipschitz assumptions in the $x$-variable. The situation in which the uniqueness of the solutions for the initial problems is not ensured can be treated as well by a standard approximation technique described in [11]. However, the conclusion of some results should be slightly modified with respect to the number of the periodic solutions we find (see Remark 5 in Section 8).

The following results can be obtained under the assumption of a locally lipschitz property of $w(t, x)$ and $g(x)$ in the $x$-variable. We suppose also that $g: J \rightarrow \mathbb{R}$ is continuously differentiable in a neighbourhood of the endpoints of its domain of definition.

Theorem 1. Let $g:\left(-\infty, b\left[\rightarrow \mathbb{R}\right.\right.$, with $b \leq+\infty$ and $\lim _{x \rightarrow b^{-}} G(x)=+\infty$, where $G$ is a primitive of $g$, be such that

$$
\limsup _{x \rightarrow-\infty} g(x)<+\infty, \quad \limsup _{x \rightarrow-\infty} g^{\prime}(x) \leq \alpha<+\infty \quad(\alpha \geq 0)
$$

and

$$
0<\gamma \leq \liminf _{x \rightarrow b^{-}} g^{\prime}(x) \leq \limsup _{x \rightarrow b^{-}} g^{\prime}(x) \leq \beta \leq+\infty
$$

Suppose that $k, m \in \mathbb{Z}_{0}^{+}$are co-prime integers such that

$$
\frac{2 \sqrt{\alpha} \sqrt{\beta}}{\sqrt{\alpha}+\sqrt{\beta}}<\frac{2 k \pi}{m T}<\sqrt{\gamma}
$$

Then, if $\beta<+\infty$ or $\gamma=+\infty$, equation $(E)_{s}$ has at least two solutions of minimal period $m T$ for each sufficiently large positive s. In the phase-plane such solutions rotate $k$ times around $P_{s}$ in the time interval $[0, m T[$.

Theorem 1 follows from Theorem 7 in Section 4 using Remark 4 in Section 7. More precise information in the case of harmonic solutions can be obtained from the corollaries in Section 6.1.

Theorem 2. Let $g:] a, b\left[\rightarrow \mathbb{R}\right.$, with $-\infty \leq a<b \leq+\infty$ and $\lim _{x \rightarrow b^{-}} G(x)=+\infty$, where $G$ is a primitive of $g$, be such that $\limsup _{x \rightarrow a^{+}} g(x)=+\infty$ and

$$
0<\gamma \leq \liminf _{x \rightarrow b^{-}} g^{\prime}(x) \leq \limsup _{x \rightarrow b^{-}} g^{\prime}(x) \leq \beta \leq+\infty
$$

Suppose that $k, m \in \mathbb{Z}_{0}^{+}$are co-prime integers such that

$$
0<\frac{2 k \pi}{m T}<\sqrt{\gamma} .
$$

Then, if $\beta<+\infty$ or $\gamma=+\infty$, equation $(E)_{s}$ has at least two solutions of minimal period $m T$ for each sufficiently large positive s. In the phase-plane such solutions rotate $k$ times around $P_{s}$ in the time interval $[0, m T[$.

Theorem 2 follows from Theorem 8 in Section 4 using Remark 4 in Section 7. More precise information in the case of harmonic solutions can be obtained from the corollaries in Section 6.2. 
In both Theorem 1 and Theorem 2, as well as in the subsequent corollaries, the case of a singularity for $b<+\infty$ is compatible only with the assumption $\beta=+\infty$, in view of the condition $G\left(b^{-}\right)=+\infty$. On the other hand, if $b=+\infty$, the condition $\lim _{x \rightarrow b^{-}} G(x)=+\infty$ is superfluous, as it follows directly from the fact that $\gamma>0$, which is implicitly assumed in all of our results.

The two $m T$-periodic solutions whose existence is asserted in Theorem 1 and Theorem 2 do not belong to the same periodicity class, that is, they cannot be obtained, one from the other, as a shift of $i T$ in time, for $1 \leq i \leq m-1$.

As mentioned above, the minimality of the period is meant in the class of the periods $j T$, with $j \in \mathbb{Z}_{0}^{+}$. This definition of subharmonic solutions of order $m$ corresponds to that considered in [38] and was used in [11], [12], [13]. On the other hand, note that this is not exactly the definition of subharmonic solutions as considered for instance in [33]. Indeed, under our assumptions, we are not able to prove that the $m T$-periodic solutions we find have $m T$ as their minimal period. This difficulty usually occurs also under different approaches for the solvability of $(E)_{s}$ or related equations (see, e.g. [34], [20]). In these cases and in order to guarantee the minimality of the period, some further assumption on $w$ is usually needed. We recall from [34] and [20] the following condition:

$\left(W^{*}\right) \quad$ If $x(t)$ is a periodic function with minimal period $q T$, with $q>0$ rational and $g(x(t))-w(t, x(t))$ is a periodic function with minimal period $q T$, then $q$ is necessarily an integer.

Under $\left(W^{*}\right)$, the subharmonic solutions we find in all our results have precisely $m T$ as minimal period. As discussed in [34] and [20], condition $\left(W^{*}\right)$ is satisfied under a wide choice of hypotheses on $w(t, x)$. For example, note that if $w=w(t)$, is a periodic function with minimal period $T>0$, then $\left(W^{*}\right)$ holds trivially. We also observe that with respect to analogous results about subharmonics obtained in [34], [17], [20], [19], where condition $\left(W^{*}\right)$ is assumed (at least implicitly), we don't require any special restriction on the integer $m$.

The case $\beta=+\infty$ and $\gamma<+\infty$ can be analysed as well and the corresponding results are presented in Theorem 9 of Section 6 and Theorem 10 of Section 7 .

In the case $\gamma=\beta=+\infty$, Theorem 1 allows any choice of $k$ and $m$ co-primes such that

$$
\frac{k}{m}>\frac{T \sqrt{\alpha}}{\pi}
$$

and Theorem 2 is valid for any choice of $k$ and $m$ co-primes. Thus, for $m=1$, and taking into account the different numbers of rotation of the solutions in the interval $[0, T$ [ for different $k$, we immediately obtain an improved version of Zinner's theorem for the periodic case which reads as follows:

Corollary 1. Let $g$ be of class $\mathcal{C}^{1}$ on $(-\infty, b[$, with $b \leq+\infty$ and such that $\lim _{x \rightarrow b^{-}} G(x)=\lim _{x \rightarrow b^{-}} g^{\prime}(x)=+\infty$. Suppose that either $\limsup _{x \rightarrow-\infty} g(x)<+\infty$ and $\limsup _{x \rightarrow-\infty} g^{\prime}(x) \leq \alpha<+\infty$, or $\limsup _{x \rightarrow-\infty} g(x)=+\infty$. Then, for each $n$ there is a constant $s_{n}$ such that for $s>s_{n},(E)_{s}$ has at least $2 n$ harmonic solutions.

Notice that here we can deal with $b<+\infty$ (the case of a singularity in $b$ ), while, if $b=+\infty$, the condition on $G(x)$ is always satisfied (as remarked above). Under the assumptions $G\left(b^{-}\right)=g^{\prime}\left(b^{-}\right)=+\infty$ of Corollary 1 we have that $\lim _{x \rightarrow b^{-}} g(x)=$ $+\infty$ as well. Concerning the behaviour of $g$ at the left end of its domain, we observe 
that when $\lim \sup _{x \rightarrow-\infty} g(x)=+\infty$, a further negative solution can be found via the method of upper and lower solutions as in Corollary 8. As a typical application of this result, we have that for each $n \in \mathbb{Z}_{0}^{+}$, there is $s_{n}>0$ such that the equation

$$
x^{\prime \prime}+x^{2}=s+w(t),
$$

with $w(\cdot)$ a $T$-periodic function, has at least $2 n+1$ harmonic solutions for $s>s_{n}$ (Example 1 in Section 8).

In the case $\beta<+\infty$, we can compare Theorem 1 with the corresponding result in $[8$, Theorem 1.2 (a)]. Indeed, our theorem for $m=1$ and $J=\mathbb{R}$ (as considered in [8]) reads now as follows:

Corollary 2. Let $g: \mathbb{R} \rightarrow \mathbb{R}$ be of class $\mathcal{C}^{1}$ and such that $\lim \sup _{x \rightarrow-\infty} g(x)<+\infty$. Assume

$$
\limsup _{x \rightarrow-\infty} g^{\prime}(x) \leq \alpha<\gamma \leq \liminf _{x \rightarrow+\infty} g^{\prime}(x) \leq \limsup _{x \rightarrow+\infty} g^{\prime}(x) \leq \beta \leq+\infty,
$$

with $\alpha \geq 0$ and let $l$ be the integer part of $(T / \pi)(\sqrt{\alpha \beta} /(\sqrt{\alpha}+\sqrt{\beta}))$. If there exists an integer $n>l$ such that $\lambda_{n}<\gamma$, then equation $(E)_{s}$ has at least $2(n-l)$ harmonic solutions for each sufficiently large positive $s$.

This last statement corresponds to Corollary 7 in Section 6.1. In the comparison between this result and the del Pino, Manásevich and Murua theorem, a slight difference is evident. Actually, in [8, Theorem 1.2 (a)] one more solution appears. This is due to the fact that in [8] the nonresonance condition $\gamma=\beta \neq \lambda_{j}$ for all $j \in \mathbb{Z}_{0}^{+}$is assumed. Indeed, we could prove the existence of that extra solution if we suppose that the interval $[\gamma, \beta]$ has empty intersection with the spectrum $\left\{\lambda_{j}\right\}$ of the differential operator $u \rightarrow-u^{\prime \prime}$ with the $T$-periodic conditions. However we have not required such an assumption in our theorem and, moreover, we have not required here the existence of limits for $g^{\prime}(x)$ at $\pm \infty$ as in [8]. See Section 6 for a more detailed discussion on this line.

From Theorem 2 with $a>-\infty$, we can obtain a simple consequence in the case of the double singularity. Indeed, if we assume that there is a singularity of attractive type at $x=a$ and of repulsive type at $x=b$, so that we can take $\gamma=+\infty$, then we can state a result as follows:

Corollary 3. Let $g$ be of class $\mathcal{C}^{1}$ on the bounded interval $] a, b[$ and assume that

$$
\limsup _{x \rightarrow a^{+}} g(x)=\lim _{x \rightarrow b^{-}} G(x)=\lim _{x \rightarrow b^{-}} g^{\prime}(x)=+\infty,
$$

where $G$ is a primitive of $g$. Then for each $m, n \in \mathbb{Z}_{0}^{+}$, there is $s_{m, n}$ such that for each $s>s_{m, n}$, equation $(E)_{s}$ has at least $2 n$ subharmonic solutions of order $m$. Therefore, the number of $m$-th order subharmonics tends to infinity as $s \rightarrow+\infty$.

This statement is a particular case of Theorem 10. Note that a similar result holds true by interchanging suitably the role of $a$ and $b$ in the limits (see Section 8).

This last result has a direct application to the study of the dynamics of a charged particle moving on a line segment at which ends one has placed two fixed charges of different sign (Example 3 in Section 8).

Finally, as shown in Section 7, it is possible to obtain a theorem about the existence of an arbitrarily large number of subharmonics of any sufficiently large order. Actually we have: 
Theorem 3. Let $g:\left(-\infty, b\left[\rightarrow \mathbb{R}\right.\right.$, with $b \leq+\infty$ and $\lim _{x \rightarrow b^{-}} G(x)=+\infty$, be such that

$$
\limsup _{x \rightarrow-\infty} g(x)<+\infty, \quad \limsup _{x \rightarrow-\infty} g^{\prime}(x) \leq \alpha,
$$

with $\alpha \geq 0$,

$$
0<\gamma \leq \liminf _{x \rightarrow b^{-}} g^{\prime}(x) \leq \limsup _{x \rightarrow b^{-}} g^{\prime}(x) \leq \beta \leq+\infty
$$

and

$$
\frac{2 \sqrt{\alpha} \sqrt{\beta}}{\sqrt{\alpha}+\sqrt{\beta}}<\sqrt{\gamma}
$$

If $\gamma<+\infty$, then for each $k \in \mathbb{Z}_{0}^{+}$there exists $m_{k}$ such that for every $m \geq m_{k},(E)_{s}$ has at least $2 k$ subharmonic solutions of order $m$ that do not belong to the same periodicity class, for each sufficiently large positive s. The same conclusion holds taking $\gamma>0$ and no restriction on $\alpha, \beta$ and $g^{\prime}(x)$ at $-\infty$, if $\lim \sup _{x \rightarrow-\infty} g(x)=$ $+\infty$.

This result is precisely a subcase of Theorem 10 of Section 7. Sharper conclusions can be obtained when $\gamma=+\infty$. In this case, for any $m>1$, we can find arbitrarily many subharmonics of order $m$ provided that $s$ is large enough.

A straightforward consequence of Theorem 3 (and Theorem 10) for the case ]$a, b[=(-\infty,+\infty)$ is the following:

Corollary 4. Let $g: \mathbb{R} \rightarrow \mathbb{R}$ of class $\mathcal{C}^{1}$ be such that the limits

$$
g^{\prime}(-\infty)=\lim _{x \rightarrow-\infty} g^{\prime}(x), \quad \text { and } g^{\prime}(+\infty)=\lim _{x \rightarrow+\infty} g^{\prime}(x)
$$

exist with

$$
g^{\prime}(-\infty)<g^{\prime}(+\infty) \quad \text { and } 0<g^{\prime}(+\infty) \leq+\infty .
$$

If $g^{\prime}(+\infty)<+\infty$, then for each $k \in \mathbb{Z}_{0}^{+}$there exists $m_{k}$ such that for every $m \geq m_{k}$, $(E)_{s}$ has at least $2 k$ subharmonic solutions of order $m$ that do not belong to the same periodicity class, for each sufficiently large positive s. If $g^{\prime}(+\infty)=+\infty$, the same conclusion holds taking $m_{k}=1$ for each $k$.

This result, in the case $0<g^{\prime}(-\infty)<g^{\prime}(+\infty)<+\infty$, applies to the study of the interference of the nonlinearity with the so-called "Dancer - Fučik spectrum" (see [6], [21]). Indeed, consider, as a special case, the piecewise linear function $g(x)=\mu x^{+}-\nu x^{-}$, with $0<\nu<\mu$, where $x^{+}=\max \{x, 0\}$ and $x^{-}=\max \{-x, 0\}$. In this connection, recall that in [26], Lazer and McKenna proved that for

$$
(j-1)^{2}<\nu \leq \mu<(j+1)^{2} \text { and } \frac{2 \sqrt{\mu} \sqrt{\nu}}{\sqrt{\mu}+\sqrt{\nu}}=j \in \mathbb{Z}_{0}^{+},
$$

the equation

$$
x^{\prime \prime}+\mu x^{+}-\nu x^{-}=A \sin (j t+\theta), \quad \text { with } A>0, \theta \in \mathbb{R},
$$

has no bounded solutions (and therefore, no periodic solutions of any period). Now, since the function $A \sin (j t+\theta)$ is periodic of minimal period $(2 \pi / j)$, according to our result, we have that the equation

$$
x^{\prime \prime}+\mu x^{+}-\nu x^{-}=s+A \sin (j t+\theta),
$$


with $0<\nu<\mu$, has an arbitrarily large number of periodic solutions of minimal period $2 n \pi$ for $n$ large, as $s \rightarrow+\infty$. The fact that $g(x)=\mu x^{+}-\nu x^{-}$is not differentiable at $x=0$ does not affect this example since Corollary 4 is true for $g$ locally lipschitzian in $\mathbb{R}$ and smooth for $|x|$ large as well.

Note that a similar conclusion holds for $\nu>\mu$ and $s$ negative with $|s|$ large, according to Theorem 12 in Section 8 , while there are no bounded solutions if $\mu=\nu$ is an eigenvalue (the resonance case).

Further remarks, corollaries and examples are collected in Sections 6 and 8.

Throughout the article we denote by $\mathbb{Z}_{0}^{+}$and $\mathbb{Z}^{+}=\mathbb{Z}_{0}^{+} \cup\{0\}$ the sets of positive and nonnegative integers, respectively. The same convention is used to define the sets $\mathbb{R}_{0}^{+}$and $\mathbb{R}^{+}$of positive and nonnegative real numbers.

\section{ACKNOWLEDGEMENT}

This work is part of C. Rebelo's Ph.D. program [39] supported by a fellowship from Programa Ciência. The authors thank the International School for Advanced Studies in Trieste (SISSA) for the kind hospitality.

\section{Polar Systems And Rotation numbers}

Let

$$
z^{\prime}=Z(t, z)
$$

be a planar system, where $Z: \mathbb{R} \times \mathcal{D} \rightarrow \mathbb{R}^{2}$ is a continuous function with the $z$-variable belonging to an open simply connected domain $\mathcal{D} \subset \mathbb{R}^{2}$.

Let $P \in \mathcal{D}$ be fixed and suppose that $z(\cdot)$ is a solution of (1) defined on a compact interval $I$, such that $z(t) \neq P$, for all $t \in I$. The use of suitable polar coordinates with respect to the point $P$, in order to represent the variable point $z(t)$, is a useful tool in the study of the qualitative behaviour of the solutions. In particular, it allows the definition of a rotation number which evaluates the number of turns of $z(t)$ around the point $P$. The most classical situation is described by the Prüfer transformation, for $\mathcal{D}=\mathbb{R}^{2}$ and $P=\mathcal{O}=(0,0)$, via the standard polar coordinates system. There are, however, cases in which the choice of different coordinates yields some substantial simplifications in the computations. Examples in this direction can be found in [4], [14], [37], [41], [42]. The aim of this section is to present a transformation which unifies the approaches in [4], [14] and works in the case where $\mathcal{D}=] A, B[\times \mathbb{R}$ is a vertical strip, $P=(c, 0)$ and, for $z=(x, y)$, (1) takes the form $x^{\prime}=y, y^{\prime}=-h(t, x)$. We use a change of variables of the form $\psi(x)=r \cos \phi, y=r \sin \phi$, with $\psi$ a suitable increasing homeomorphism. More general transformations like $\psi(x)=r \cos \phi, \zeta(y)=r \sin \phi$ could be considered as well without significant changes in the discussion.

Definition. We say that an increasing homeomorphism $\psi$ from $] A, B$ [ onto $\mathbb{R}$, with $-\infty \leq A<B \leq+\infty$, is admissible with respect to $c \in] A, B$ [if $\psi$ is of class $\mathcal{C}^{1}$ in ]$A, B[\backslash\{c\}$ with $\psi(c)=0$.

In this case $\psi(x)(x-c)>0, \forall x \neq c, \lim _{x \rightarrow A^{+}} \psi(x)=-\infty$ and $\lim _{x \rightarrow B^{-}} \psi(x)=$ $+\infty$. For each function $\psi:] A, B[\rightarrow \mathbb{R}$, admissible with respect to some $c \in] A, B$, we will denote by $\left.\Pi_{\psi}: \mathbb{R}_{0}^{+} \times \mathbb{R} \rightarrow \mathcal{D} \backslash\{P\}=\right] A, B[\times \mathbb{R} \backslash\{P\}$ the map defined by $\Pi_{\psi}(r, \phi)=\left(\psi^{-1}(r \cos \phi), r \sin \phi\right)$. Note that, according to $[31], E_{\psi}=\left(\mathbb{R}_{0}^{+} \times \mathbb{R}, \Pi_{\psi}\right)$ 
is a covering space of $] A, B[\times \mathbb{R} \backslash\{P\}$. When $] A, B[=\mathbb{R}$, a typical function which is admissible with respect to $c$ is given by $\psi(x)=x-c$. In this case $\Pi_{\psi}(r, \phi)=$ $(c+r \cos \phi, r \sin \phi)$ and thus for a point $(x, y) \in \mathbb{R}^{2} \backslash\{P\}$ the corresponding $r$ and $\phi$ are its polar coordinates with center in $P$.

Let $\psi:] A, B[\rightarrow \mathbb{R}$ be an admissible function with respect to some $c \in] A, B[$ and consider the covering space $E_{\psi}$ of $] A, B[\times \mathbb{R} \backslash\{P\}$. Let $z(\cdot)$ be a continuous function defined on a compact interval $I$ such that $z(t) \in] A, B[\times \mathbb{R} \backslash\{P\}, \forall t \in I$ and denote by $\left(r_{\psi}, \phi_{\psi}\right)$ a lifting of $z(\cdot)$ to $E_{\psi}$.

Let $s_{1}, s_{2} \in I$. We wish to define the rotation of the function $z(\cdot)$ around the point $P$, from $t=s_{1}$ to $t=s_{2}$ as the angular change in the clockwise sense for $t$ in the oriented interval $\left[s_{1}, s_{2}\right]$. Accordingly, we introduce the following definition.

Definition. The rotation number of $z(\cdot)$ from $s_{1}$ to $s_{2}$, with respect to the coordinate system given by $E_{\psi}$, denoted by $\operatorname{rot}_{\psi}\left(s_{2}, s_{1} ; z\right)$, is

$$
\operatorname{rot}_{\psi}\left(s_{2}, s_{1} ; z\right)=\frac{\phi_{\psi}\left(s_{1}\right)-\phi_{\psi}\left(s_{2}\right)}{2 \pi} .
$$

We observe that, given $\psi$, this number does not depend on the lifting $\left(r_{\psi}, \phi_{\psi}\right)$ chosen.

In the case that $\psi(x)=x-c$, we denote $\operatorname{rot}_{\psi}\left(s_{2}, s_{1} ; z\right)$ by $\operatorname{rot}\left(s_{2}, s_{1} ; z\right)$.

It is our interest to find some relations between the rotation numbers with respect to two different systems of coordinates. To this end we will establish some preliminary lemmas first.

In what follows, $\left.\psi_{1}:\right] A_{1}, B_{1}\left[\rightarrow \mathbb{R}\right.$ and $\left.\psi_{2}:\right] A_{2}, B_{2}[\rightarrow \mathbb{R}$ will be admissible functions with respect to the same $c \in] A_{1}, B_{1}[\cap] A_{2}, B_{2}\left[\right.$ and $E_{\psi_{1}}, E_{\psi_{2}}$ the corresponding covering spaces of $] A_{1}, B_{1}[\times \mathbb{R} \backslash\{P\}$ and $] A_{2}, B_{2}[\times \mathbb{R} \backslash\{P\}$ respectively. Suppose that $z(\cdot)=(x(\cdot), y(\cdot))$ is a continuous function defined on a compact interval $I$, such that $z(t) \in \mathcal{D} \backslash\{P\}=(] A_{1}, B_{1}[\cap] A_{2}, B_{2}[) \times \mathbb{R} \backslash\{P\}, \forall t \in I$. Consider some liftings $\left(r_{\psi_{1}}(\cdot), \phi_{\psi_{1}}(\cdot)\right)$ and $\left(r_{\psi_{2}}(\cdot), \phi_{\psi_{2}}(\cdot)\right)$ of $z(\cdot)$ to $E_{\psi_{1}}$ and $E_{\psi_{2}}$, respectively.

Lemma 1. Let $k \in \mathbb{Z}$. If there are $t_{0} \in I$ and $i \in\{1,2\}$ with $\phi_{\psi_{i}}\left(t_{0}\right)=k \frac{\pi}{2}$, then

$$
\frac{2}{\pi}\left(\phi_{\psi_{2}}\left(t_{0}\right)-\phi_{\psi_{1}}\left(t_{0}\right)\right) \equiv 0(\bmod 4)
$$

Lemma 2. Let $t_{0} \in I$ be such that $\phi_{\psi_{1}}\left(t_{0}\right)=k \frac{\pi}{2}$, for some $k \in \mathbb{Z}$. Then we can choose a lifting $\left(r_{\psi_{2}}(\cdot), \phi_{\psi_{2}}(\cdot)\right)$ to $E_{\psi_{2}}$ such that $\phi_{\psi_{1}}\left(t_{0}\right)=\phi_{\psi_{2}}\left(t_{0}\right)$.

The proof of Lemma 1 and Lemma 2 is an easy consequence of the definitions and therefore it is omitted.

Lemma 3. Suppose that there exist $t_{0} \in I$ and $k \in \mathbb{Z}$ such that $\phi_{\psi_{1}}\left(t_{0}\right)=\phi_{\psi_{2}}\left(t_{0}\right)=$ $k \frac{\pi}{2}$. Then, for any $s \in \mathbb{Z}$ and $t \in I$,

$$
\phi_{\psi_{1}}(t)=s \frac{\pi}{2} \Longleftrightarrow \phi_{\psi_{2}}(t)=s \frac{\pi}{2} .
$$

Proof. By contradiction, suppose there exists a $t \neq t_{0}$ such that $\phi_{\psi_{1}}(t)=s \frac{\pi}{2}$ and $\phi_{\psi_{2}}(t)=r \frac{\pi}{2}$ for some $r, s \in \mathbb{Z}, r \neq s$. Without loss of generality, we assume $t>t_{0}$ 
and left out the case $t<t_{0}$ which is completely similar. Denote by $t_{2}$ the minimal of the $t>t_{0}$ for which the above condition holds. Thus we have $\phi_{\psi_{1}}\left(t_{2}\right)=s^{*} \frac{\pi}{2}$, $\phi_{\psi_{2}}\left(t_{2}\right)=r^{*} \frac{\pi}{2}$ for some $r^{*}, s^{*} \in \mathbb{Z}, r^{*} \neq s^{*}$. Moreover, by the minimality of $t_{2}$, if for some $t \in\left[t_{0}, t_{2}\left[\right.\right.$ and for some $l \in \mathbb{Z}, \phi_{\psi_{1}}(t)=l \frac{\pi}{2}$, then $\phi_{\psi_{2}}(t)=l \frac{\pi}{2}$ too. Let $t_{1} \in\left[t_{0}, t_{2}\right.$ [ be the maximal of the $t \in\left[t_{0}, t_{2}\right]$ such that $\frac{2}{\pi} \phi_{\psi_{1}}(t)=\frac{2}{\pi} \phi_{\psi_{2}}(t) \in \mathbb{Z}$ and set $m=\frac{2}{\pi} \phi_{\psi_{1}}\left(t_{1}\right)=\frac{2}{\pi} \phi_{\psi_{2}}\left(t_{1}\right)$. Using Lemma 1 we observe that by the maximality of $t_{1}$ and the minimality of $t_{2}$, we have that $\frac{2}{\pi} \phi_{\psi_{i}}(t) \notin \mathbb{Z}$, for all $\left.t \in\right] t_{1}, t_{2}[$. Hence, by the continuity of $\phi_{\psi_{1}}$, we conclude that $s^{*}=m+1, s^{*}=m$ or $s^{*}=m-1$ and also, by the continuity of $\phi_{\psi_{2}}$, we have that $r^{*}=m+1, r^{*}=m$ or $r^{*}=m-1$. An elementary analysis of all the possibilities shows that if $s^{*} \neq r^{*}$, then, in any case, $s^{*} \not \equiv r^{*}(\bmod 4)$. This contradicts Lemma 1.

Lemma 4. We have the following properties:

i) If there exist $t_{0} \in I, k \in \mathbb{Z}$ and $\varepsilon>0$ such that $\phi_{\psi_{1}}\left(t_{0}\right)=k \frac{\pi}{2}$ and $(k-1) \frac{\pi}{2}<$ $\left.\phi_{\psi_{1}}(t)<k \frac{\pi}{2}, \forall t \in\right] t_{0}, t_{0}+\varepsilon[$, then the same property is satisfied by a suitable choice of $\phi_{\psi_{2}}$.

ii) If there exist $t_{0} \in I, k \in \mathbb{Z}$ and $\varepsilon>0$ such that $\phi_{\psi_{1}}\left(t_{0}\right)=k \frac{\pi}{2}$ and $(k+1) \frac{\pi}{2}>$ $\left.\phi_{\psi_{1}}(t)>k \frac{\pi}{2}, \forall t \in\right] t_{0}-\varepsilon, t_{0}[$, then the same property is satisfied by a suitable choice of $\phi_{\psi_{2}}$.

iii) If for some $t_{0} \in I, k \in \mathbb{Z}$ and $\varepsilon>0$ we have $(k-1) \frac{\pi}{2}<\phi_{\psi_{1}}(t)<k \frac{\pi}{2}, \forall t \in$ ]$t_{0}, t_{0}+\varepsilon\left[\right.$ and $\left.(k+1) \frac{\pi}{2}>\phi_{\psi_{1}}(t)>k \frac{\pi}{2}, \forall t \in\right] t_{0}-\varepsilon, t_{0}[$, then the same property is satisfied by a suitable choice of $\phi_{\psi_{2}}$.

These properties remain valid if we replace $] t_{0}, t_{0}+\varepsilon[$ by $] t_{0}-\varepsilon, t_{0}[$ and vice-versa.

Proof. Let us prove i). By Lemma 2 we can choose a lifting $\left(r_{\psi_{2}}(\cdot), \phi_{\psi_{2}}(\cdot)\right)$ to $E_{\psi_{2}}$ such that $\phi_{\psi_{2}}\left(t_{0}\right)=k \frac{\pi}{2}$. Let $(x(t), y(t))=\Pi_{\psi_{1}}\left(r_{\psi_{1}}(t), \phi_{\psi_{1}}(t)\right)$. Suppose $k \equiv 1(\bmod 4)$. Then, as $(k-1) \frac{\pi}{2}<\phi_{\psi_{1}}(t)<k \frac{\pi}{2}$, we have $\cos \phi_{\psi_{1}}(t)>0$ and $\sin \phi_{\psi_{1}}(t)>0$ for all $\left.t \in\right] t_{0}, t_{0}+\varepsilon[$. Hence

$$
x(t)=\psi_{1}^{-1}\left(r_{\psi_{1}}(t) \cos \phi_{\psi_{1}}(t)\right)>c \quad \text { and } \quad y(t)=r_{\psi_{1}}(t) \sin \phi_{\psi_{1}}(t)>0
$$

from which we conclude that there exists $m \in \mathbb{Z}$ such that $(k-1) \frac{\pi}{2}+2 m \pi<$ $\phi_{\psi_{2}}(t)<k \frac{\pi}{2}+2 m \pi$ for every $\left.t \in\right] t_{0}, t_{0}+\varepsilon[$. The result follows now from the fact that $\phi_{\psi_{2}}$ is a continuous function and $\phi_{\psi_{2}}\left(t_{0}\right)=k \frac{\pi}{2}$. The remaining cases for $k$ can be treated in a similar way.

The proof of ii) is analogous to that of i). In order to prove iii) first notice that by continuity $\phi_{\psi_{1}}\left(t_{0}\right)=k \frac{\pi}{2}$. Then we can apply i) and ii) and find two liftings $\left(r_{\psi_{2}}(\cdot), \phi_{\psi_{2}}(\cdot)\right)$ and $\left(r_{\psi_{2}}^{*}(\cdot), \phi_{\psi_{2}}^{*}(\cdot)\right)$ to $E_{\psi_{2}}$ satisfying $\phi_{\psi_{2}}\left(t_{0}\right)=k \frac{\pi}{2},(k-1) \frac{\pi}{2}<$ $\left.\phi_{\psi_{2}}(t)<k \frac{\pi}{2}, \forall t \in\right] t_{0}, t_{0}+\varepsilon\left[\right.$ and $\phi_{\psi_{2}}^{*}\left(t_{0}\right)=k \frac{\pi}{2},(k+1) \frac{\pi}{2}>\phi_{\psi_{2}}^{*}(t)>k \frac{\pi}{2}, \forall t \in$ ]$t_{0}-\varepsilon, t_{0}[$. Two liftings of the same function that coincide at one point must coincide, so $\phi_{\psi_{2}} \equiv \phi_{\psi_{2}}^{*}$ and this concludes the proof.

The same argument applies if we change the role of the two intervals.

Lemma 5. Let $\sigma_{1}<\sigma_{2}$ with $\sigma_{1}, \sigma_{2} \in I$. Suppose that there exists $t_{0} \in\left[\sigma_{1}, \sigma_{2}\right]$ such that $\phi_{\psi_{1}}\left(t_{0}\right)=k \frac{\pi}{2}$ for some $k \in \mathbb{Z}$ and one of the following inequalities holds:

i) $\left(\phi_{\psi_{1}}(t)-k \frac{\pi}{2}\right)\left(t-t_{0}\right)>0, \forall t \in\left[\sigma_{1}, \sigma_{2}\right] \backslash\left\{t_{0}\right\}$;

ii) $\left(\phi_{\psi_{1}}(t)-k \frac{\pi}{2}\right)\left(t-t_{0}\right)<0, \forall t \in\left[\sigma_{1}, \sigma_{2}\right] \backslash\left\{t_{0}\right\}$.

Then, for any admissible function $\psi_{2}$ we have that $\operatorname{rot}_{\psi_{2}}\left(\sigma_{2}, \sigma_{1} ; z\right)<0$ when i) is satisfied and $\operatorname{rot}_{\psi_{2}}\left(\sigma_{2}, \sigma_{1} ; z\right)>0$ when ii) is satisfied. 
Proof. Suppose that i) is satisfied with $\left.t_{0} \in\right] \sigma_{1}, \sigma_{2}\left[\right.$. By the continuity of $\phi_{\psi_{1}}$ and by $\phi_{\psi_{1}}\left(t_{0}\right)=k \frac{\pi}{2}$, we can choose a sufficiently small $\varepsilon>0$ such that $(k-1) \frac{\pi}{2}<\phi_{\psi_{1}}(t)<$ $\left.k \frac{\pi}{2}, \forall t \in\right] t_{0}-\varepsilon, t_{0}\left[\right.$, and $\left.(k+1) \frac{\pi}{2}>\phi_{\psi_{1}}(t)>k \frac{\pi}{2}, \forall t \in\right] t_{0}, t_{0}+\varepsilon\left[\right.$. Let $\psi_{2}$ be an arbitrary admissible function with respect to $c$. We can apply Lemma 4 and conclude the existence of a lifting of $z(\cdot)$ to $E_{\psi_{2}},\left(r_{\psi_{2}}(\cdot), \phi_{\psi_{2}}(\cdot)\right)$ such that $\phi_{\psi_{2}}\left(t_{0}\right)=k \frac{\pi}{2}$, $\left.(k-1) \frac{\pi}{2}<\phi_{\psi_{2}}(t)<k \frac{\pi}{2}, \forall t \in\right] t_{0}-\varepsilon, t_{0}\left[\right.$, and $\left.(k+1) \frac{\pi}{2}>\phi_{\psi_{2}}(t)>k \frac{\pi}{2}, \forall t \in\right] t_{0}, t_{0}+\varepsilon[$. By Lemma 3, as $\phi_{\psi_{1}}(t) \neq k \frac{\pi}{2}, \forall t \in\left[\sigma_{1}, \sigma_{2}\right] \backslash\left\{t_{0}\right\}$, the same is true for $\phi_{\psi_{2}}(t)$ and thus we conclude that $\phi_{\psi_{2}}(t)<k \frac{\pi}{2}, \forall t \in\left[\sigma_{1}, t_{0}\left[\right.\right.$ and $\left.\left.\phi_{\psi_{2}}(t)>k \frac{\pi}{2}, \forall t \in\right] t_{0}, \sigma_{2}\right]$. Therefore, we finally obtain $\operatorname{rot}_{\psi_{2}}\left(\sigma_{2}, \sigma_{1} ; z\right)=\frac{\phi_{\psi_{2}}\left(\sigma_{1}\right)-\phi_{\psi_{2}}\left(\sigma_{2}\right)}{2 \pi}<0$. Note that, as said before, the rotation does not depend on the particular lifting. The case ii) as well as $t_{0}=\sigma_{1}$ or $t_{0}=\sigma_{2}$ can be treated similarly.

Theorem 4. For each pair of admissible functions $\left(\psi_{1}, \psi_{2}\right)$ there exists a constant $\delta=\delta_{\left(\psi_{1}, \psi_{2}\right)}$ with $0 \leq \delta \leq 1 / 4$, such that for every $k \in \mathbb{Z}$, every continuous function $z(\cdot)$, with $z(t) \in \mathcal{D} \backslash\{P\} \forall t \in I$ and any $s_{1}, s_{2} \in I$, we have

$$
\begin{aligned}
& \operatorname{rot}_{\psi_{1}}\left(s_{2}, s_{1} ; z\right)>k+\delta \Longrightarrow \operatorname{rot}_{\psi_{2}}\left(s_{2}, s_{1} ; z\right)>k \\
& \operatorname{rot}_{\psi_{2}}\left(s_{2}, s_{1} ; z\right)>k+\delta \Longrightarrow \operatorname{rot}_{\psi_{1}}\left(s_{2}, s_{1} ; z\right)>k
\end{aligned}
$$

and

$$
\begin{gathered}
\operatorname{rot}_{\psi_{1}}\left(s_{2}, s_{1} ; z\right)<k-\delta \Longrightarrow \operatorname{rot}_{\psi_{2}}\left(s_{2}, s_{1} ; z\right)<k \\
\operatorname{rot}_{\psi_{2}}\left(s_{2}, s_{1} ; z\right)<k-\delta \Longrightarrow \operatorname{rot}_{\psi_{1}}\left(s_{2}, s_{1} ; z\right)<k
\end{gathered}
$$

Proof. For sake of conciseness we give a proof only for the cases $(2),\left(2^{*}\right)$ with $s_{1}<s_{2}$ and $k \in \mathbb{Z}^{+}$. Actually this is the only situation which is used in the sequel of our paper. See [39] for all the missing details.

We prove that (2) holds with $\delta=1 / 4$. Assume $\operatorname{rot}_{\psi_{1}}\left(s_{2}, s_{1} ; z\right)>k+1 / 4$, for some $k \in \mathbb{Z}^{+}$and $s_{1}<s_{2}$. Let $b=\frac{2 \phi_{\psi_{1}}\left(s_{1}\right)}{\pi}$ and $a=\frac{2 \phi_{\psi_{1}}\left(s_{2}\right)}{\pi}$. By the assumption, $a<b$ and the interval $[a, b]$ has length $b-a>4 k+1$. Consider the set $[a, b] \cap \mathbb{Z}$, let $n$ be the number of its points and observe that $n \geq 4 k+1$. Let $i \in \mathbb{Z}$ be the smallest integer greater than or equal to $a$ and take the greatest $t_{2} \in\left[s_{1}, s_{2}\right]$ such that $\phi_{\psi_{1}}\left(t_{2}\right)=i \frac{\pi}{2}$. By Lemma 2, choose the lifting $\left(r_{\psi_{2}}(\cdot), \phi_{\psi_{2}}(\cdot)\right)$ of $z(\cdot)$ to $E_{\psi_{2}}$ such that $\phi_{\psi_{1}}\left(t_{2}\right)=\phi_{\psi_{2}}\left(t_{2}\right)$. Let $j \in \mathbb{Z}$ be the greatest integer smaller than or equal to $b$ and take the least $t_{1} \in\left[s_{1}, s_{2}\right]$ such that $\phi_{\psi_{1}}\left(t_{1}\right)=j \frac{\pi}{2}$. By definition, $[a, b] \cap \mathbb{Z}=[i, j] \cap \mathbb{Z}$, so that $j=i+n-1 \geq i+4 k$. By Lemma 3, $\frac{2 \phi_{\psi_{1}}\left(t_{1}\right)}{\pi}=\frac{2 \phi_{\psi_{2}}\left(t_{1}\right)}{\pi}=j$. Hence $\operatorname{rot}_{\psi_{2}}\left(t_{2}, t_{1} ; z\right)=\frac{n-1}{4}$.

We claim that $\operatorname{rot}_{\psi_{2}}\left(s_{2}, t_{2} ; z\right) \geq 0$ and $\operatorname{rot}_{\psi_{2}}\left(t_{1}, s_{1} ; z\right) \geq 0$, with strict inequalities if $s_{l} \neq t_{l}$. Indeed, this assertion is a consequence of the fact that if $s_{l}=t_{l}$ the rotation is zero, otherwise, as $\phi_{\psi_{1}}(t)<i \pi / 2$ for $\left.\left.t \in\right] t_{2}, s_{2}\right]$ and $\phi_{\psi_{1}}(t)>j \pi / 2$ for $t \in\left[s_{1}, t_{1}[\right.$, we can apply Lemma 5 in case ii) and the result follows. We can now conclude that

$$
\begin{aligned}
\operatorname{rot}_{\psi_{2}}\left(s_{2}, s_{1} ; z\right) & =\operatorname{rot}_{\psi_{2}}\left(s_{2}, t_{2} ; z\right)+\operatorname{rot}_{\psi_{2}}\left(t_{2}, t_{1} ; z\right)+\operatorname{rot}_{\psi_{2}}\left(t_{1}, s_{1} ; z\right) \\
& \geq \frac{n-1}{4} \geq k
\end{aligned}
$$


From the last inequalities, we conclude that $\operatorname{rot}_{\psi_{2}}\left(s_{2}, s_{1} ; z\right)>k$. In fact, if we suppose that $\operatorname{rot}_{\psi_{2}}\left(s_{2}, s_{1} ; z\right)=k$, then we obtain $n=4 k+1$ and $\operatorname{rot}_{\psi_{2}}\left(s_{l}, t_{l} ; z\right)=0$, for $l=1,2$. Hence by the above claim it is $s_{l}=t_{l}$ and so $a=i$ and $b=j$ from which $b-a=4 k$ follows and this contradicts the assumption $b-a>4 k+1$.

Notice that reversing the roles of $\psi_{1}$ and $\psi_{2}$ we conclude that $\left(2^{*}\right)$ is satisfied for $\delta=1 / 4$.

The constant $\delta_{\left(\psi_{1}, \psi_{2}\right)} \in[0,1 / 4]$ will be defined as the minimal $\delta \geq 0$ for which $(2),\left(2^{*}\right)$ and $(3),\left(3^{*}\right)$ are satisfied.

Notation. In what follows we will denote $\delta_{(\psi, x-c)}$ by $\delta_{\psi}$.

Remark 1. In some special cases, it is possible to prove that $\delta_{\left(\psi_{1}, \psi_{2}\right)}=0$. For example, if $\psi_{1}(x)=\mu_{1}(x-c)^{+}-\nu_{1}(x-c)^{-}$and $\psi_{2}(x)=\mu_{2}(x-c)^{+}-\nu_{2}(x-c)^{-}$, with $\mu_{i}>0, \nu_{i}>0$ for $i=1,2$, we have that $\delta_{\left(\psi_{1}, \psi_{2}\right)}=0$. Indeed, let $\psi_{1}$ and $\psi_{2}$ be as above and let $z(\cdot)$ be such that $z(t) \neq P, \forall t \in I$. Elementary computations show that $\phi_{\psi_{1}}(t)<\phi_{\psi_{1}}(s)$ if and only if $\phi_{\psi_{2}}(t)<\phi_{\psi_{2}}(s)$, provided that $|t-s|$ is small enough, and $\phi_{\psi_{1}}(t) \equiv \phi_{\psi_{1}}(s)(\bmod 2 \pi)$ if and only if $\phi_{\psi_{2}}(t) \equiv \phi_{\psi_{2}}(s)(\bmod 2 \pi)$. This latter property, in turns, implies that $\operatorname{rot}_{\psi_{1}}\left(s_{2}, s_{1} ; z\right)=k_{1} \in \mathbb{Z}$ if and only if $\operatorname{rot}_{\psi_{2}}\left(s_{2}, s_{1} ; z\right)=k_{2} \in \mathbb{Z}$. Now, from Theorem 4 , we know that $\left|k_{1}-k_{2}\right| \leq 1 / 4$ and thus $k_{1}=k_{2}$ (actually one could easily check this fact by a direct argument without invoking Theorem 4). Thus we find that the two rotations coincide when one of the two takes integer values. Now, suppose by contradiction, that $\operatorname{rot}_{\psi_{1}}\left(s_{2}, s_{1} ; z\right)<k$ and $\operatorname{rot}_{\psi_{2}}\left(s_{2}, s_{1} ; z\right) \geq k$ for some $k \in \mathbb{Z}$. In this case, take a maximal $t \in\left[s_{1}, s_{2}\right]$ such that $\operatorname{rot}_{\psi_{2}}\left(t, s_{1} ; z\right)=k$. Hence $\operatorname{rot}_{\psi_{1}}\left(t, s_{1} ; z\right)=k$ as well. Clearly, if $t=s_{2}$, we are done, since a contradiction is achieved. So, let $t<s_{2}$ and observe that $\phi_{\psi_{2}}(s)>\phi_{\psi_{2}}(t)$ and thus $\phi_{\psi_{1}}(s)>\phi_{\psi_{1}}(t)$ for $t<s \leq s_{2}, s-t$ sufficiently small. Now, for such $s, \operatorname{rot}_{\psi_{1}}\left(s, s_{1} ; z\right)>k>\operatorname{rot}_{\psi_{1}}\left(s_{2}, s_{1} ; z\right)$. Then there is a further $t<t^{*}<s_{2}$ such that $\operatorname{rot}_{\psi_{1}}\left(t^{*}, s_{1} ; z\right)=k=\operatorname{rot}_{\psi_{2}}\left(t^{*}, s_{1} ; z\right)$, which contradicts the maximality of $t$.

In this manner we have (3) of Theorem 4 satisfied for $\delta=0$. A similar argument yields the validity of $(2),\left(2^{*}\right)$ and $\left(3^{*}\right)$ with $\delta=0$. Thus $\delta_{\left(\psi_{1}, \psi_{2}\right)}=0$. As an immediate consequence we have also that $\delta_{\psi}=0$, for any $\psi(x)=\mu(x-c)^{+}-$ $\nu(x-c)^{-}$with $\mu, \nu>0$. This property is implicitly used in [14] and discussed in [42] as a particular case of an action-angle transformation with positively homogeneous potential.

Consider now the planar system

$$
\left\{\begin{array}{l}
x^{\prime}=y \\
y^{\prime}=-X(t, x)
\end{array}\right.
$$

where $X: \mathbb{R} \times] a, b[\rightarrow \mathbb{R}$ is a continuous function, $T$-periodic in the first variable and such that for each $t_{0} \in \mathbb{R}$ and each $\left.z_{0}=\left(x_{0}, y_{0}\right) \in \mathcal{S}=\right] a, b[\times \mathbb{R}$ there is an unique solution $z\left(\cdot ; t_{0}, z_{0}\right)=\left(x\left(\cdot ; t_{0}, z_{0}\right), y\left(\cdot ; t_{0}, z_{0}\right)\right)$ of $(4)$ satisfying the initial condition $z\left(t_{0}\right)=z_{0}$ with $z\left(\cdot ; t_{0}, z_{0}\right)$ globally defined on $\mathbb{R}$. Reference to $t_{0}$ will be omitted when $t_{0}=0$.

Let $\psi:] A, B[\rightarrow \mathbb{R}$ be an admissible function with respect to $c \in] a, b[\cap] A, B[$ and let $E_{\psi}$ be the corresponding covering space of $] A, B[\times \mathbb{R} \backslash\{P\}$ where, as usual, $P=(c, 0)$. Fix $m \in \mathbb{Z}_{0}^{+}$. Suppose that $z(\cdot)$ is a solution of $(4)$ such that $z(t) \in$ ]$A, B\left[\times \mathbb{R} \backslash\{P\}, \forall t \in[0, m T]\right.$ and denote by $\left(r_{\psi}(\cdot), \phi_{\psi}(\cdot)\right)$ a corresponding lifting to 
$E_{\psi}$. For $s_{1}, s_{2} \in[0, m T]$, we defined above $\operatorname{rot}_{\psi}\left(s_{2}, s_{1} ; z\right)$. In what follows, we use simplified notations for the rotation number in some particular cases. Namely, we set

$$
\operatorname{rot}_{\psi}(m T ; z):=\operatorname{rot}_{\psi}(m T, 0 ; z) .
$$

Moreover if $z(t)=z\left(t ; z_{0}\right)$ is the solution of (4) with $z(0)=z_{0}$ defined on $[0, m T]$, then we set

$$
\operatorname{rot}_{\psi}\left(m T ; z_{0}\right):=\operatorname{rot}_{\psi}\left(m T ; z\left(\cdot ; z_{0}\right)\right)=\operatorname{rot}_{\psi}\left(m T, 0 ; z\left(\cdot ; z_{0}\right)\right) .
$$

Observe that the set $\mathcal{N}=\{t \in[0, m T]: x(t)=c\}$ is finite. Indeed $x^{\prime}(t)=y(t) \neq 0$ if $x(t)=c$. Hence, any zero of $x(\cdot)-c$ is simple and also, if $t \in[0, m T]$ is such that $\phi_{\psi}(t)=\frac{\pi}{2}+i \pi$ for some $i \in \mathbb{Z}$ then $\left(\phi_{\psi}(s)-\phi_{\psi}(t)\right)(s-t)<0$ for all $s \neq t$. Therefore we can conclude that $\operatorname{rot}_{\psi}(s, t ; z)>-1 / 2$ for any $s, t \in[0, m T]$ with $s>t$. In particular the rotation over an interval $[t, s] \subset[0, m T]$ must be nonnegative when it is an integer. Notice that $(r(\cdot), \phi(\cdot))$ is of class $\mathcal{C}^{1}$ in $[0, m T] \backslash \mathcal{N}$ and on this set we have

$$
-\phi_{\psi}^{\prime}(t)=\frac{\psi^{\prime}(x(t)) y^{2}(t)+X(t, x(t)) \psi(x(t))}{\psi^{2}(x(t))+y^{2}(t)} .
$$

The next lemma concerns the rotation number of a solution $z(\cdot)$ of (4) between $s_{1}$ and $s_{2}$. We suppose $s_{1}<s_{2}$. Using the fact that $\operatorname{rot}_{\psi}\left(s_{2}, s_{1} ; z\right)=-\operatorname{rot}_{\psi}\left(s_{1}, s_{2} ; z\right)$, it can be easily seen that this result is still valid, (with the appropriate changes), in the case $s_{1} \geq s_{2}$.

Lemma 6. For any $z(\cdot)$ solution of (4) with $z(\cdot) \neq P, \forall t \in\left[s_{1}, s_{2}\right]$ we have

$$
\operatorname{rot}_{\psi}\left(s_{2}, s_{1} ; z\right)=\frac{1}{2 \pi} \int_{s_{1}}^{s_{2}} \frac{\psi^{\prime}(x(t)) y^{2}(t)+X(t, x(t)) \psi(x(t))}{\psi^{2}(x(t))+y^{2}(t)} d t .
$$

Moreover, if $\operatorname{rot}_{\psi}\left(s_{2}, s_{1} ; z\right) \in \mathbb{Z}$, then

$$
\begin{aligned}
\operatorname{rot}_{\psi}\left(s_{2}, s_{1} ; z\right) & =\frac{1}{\pi} \int_{\left\{t \in\left[s_{1}, s_{2}\right]: x(t)>c\right\}} \frac{\psi^{\prime}(x(t)) y^{2}(t)+X(t, x(t)) \psi(x(t))}{\psi^{2}(x(t))+y^{2}(t)} d t \\
& =\frac{1}{\pi} \int_{\left\{t \in\left[s_{1}, s_{2}\right]: x(t)<c\right\}} \frac{\psi^{\prime}(x(t)) y^{2}(t)+X(t, x(t)) \psi(x(t))}{\psi^{2}(x(t))+y^{2}(t)} d t .
\end{aligned}
$$

Proof. The first part of the claim is obvious if $\mathcal{N} \cap\left[s_{1}, s_{2}\left[=\emptyset\right.\right.$. Thus, let $\mathcal{N} \cap\left[s_{1}, s_{2}[=\right.$ $\left\{t_{i}, \quad i=1, \ldots, N\right\}$ and denote by $t_{0}=s_{1}$ and $t_{N+1}=s_{2}$. Then, by an easy computation we have

$$
\phi_{\psi}\left(t_{i}\right)-\phi_{\psi}\left(t_{i+1}\right)=\int_{t_{i}}^{t_{i+1}}-\phi_{\psi}^{\prime}(t) d t, \quad \forall i=0, \ldots, N .
$$

Hence

$$
\begin{aligned}
\int_{s_{1}}^{s_{2}}-\phi_{\psi}^{\prime}(t) d t & =\sum_{i=0}^{N} \int_{t_{i}}^{t_{i+1}}-\phi_{\psi}^{\prime}(t) d t \\
& =\sum_{i=0}^{N}\left(\phi_{\psi}\left(t_{i}\right)-\phi_{\psi}\left(t_{i+1}\right)\right),
\end{aligned}
$$


from which the first part of the lemma follows using (5).

Assume now that $\operatorname{rot}_{\psi}\left(s_{2}, s_{1} ; z\right)=k \in \mathbb{Z}$. As we observed above, $k \in \mathbb{Z}^{+}$and the zeros of $x(\cdot)-c$ are simple. Thus, excluding the obvious situation when $k=0$, we have $N=2 k$ and $s_{1}=t_{0} \leq t_{1}<\ldots<t_{2 k}<t_{2 k+1}=s_{2}$.

Suppose, in order to fix the ideas, that $\phi_{\psi}\left(t_{1}\right)=\frac{\pi}{2}+2 j \pi$, where $j \in \mathbb{Z}$. This means that $x\left(t_{1}\right)=c$ with $x^{\prime}\left(t_{1}\right)=y\left(t_{1}\right)>0$. Hence $x(t)>c$ for $\left.t \in\right] t_{1}, t_{2}[$ and $x^{\prime}\left(t_{2}\right)<0$. Proceeding further, we have that $x(t)<c$ for each $\left.t \in\right] t_{i}, t_{i+1}[$ with $i$ even and $x(t)>c$ for each $t \in] t_{i}, t_{i+1}$ [ with $i$ odd. Consequently we obtain

$$
\begin{aligned}
\frac{1}{\pi} \int_{\left\{t \in\left[s_{1}, s_{2}\right]: x(t)>c\right\}}-\phi_{\psi}^{\prime}(t) d t & =\frac{1}{\pi} \sum_{i \text { odd }} \int_{t_{i}}^{t_{i+1}}-\phi_{\psi}^{\prime}(t) d t \\
& =\frac{1}{\pi}(k \pi)=\operatorname{rot}_{\psi}\left(s_{2}, s_{1} ; z\right) .
\end{aligned}
$$

On the other hand

$k=\operatorname{rot}_{\psi}\left(s_{2}, s_{1} ; z\right)=\frac{1}{2 \pi} \int_{s_{1}}^{s_{2}}-\phi_{\psi}^{\prime}(t) d t=\frac{1}{2 \pi}\left(k \pi+\int_{\left\{t \in\left[s_{1}, s_{2}\right]: x(t)<c\right\}}-\phi_{\psi}^{\prime}(t) d t\right)$,

thus

$$
\frac{1}{\pi} \int_{\left\{t \in\left[s_{1}, s_{2}\right]: x(t)<c\right\}}-\phi_{\psi}^{\prime}(t) d t=k
$$

and the result follows. The case when $\phi_{\psi}\left(t_{1}\right)=\frac{3 \pi}{2}+2 j \pi$ for some $j \in \mathbb{Z}$ can be treated similarly.

Remark 1 (continued). In the case when

$$
\psi(x)=\mu(x-c)^{+}-\nu(x-c)^{-}, \mu, \nu>0,
$$

the second claim in Lemma 6 reads as

$$
\begin{aligned}
\operatorname{rot}_{\psi}\left(s_{2}, s_{1} ; z\right) & =\frac{\nu}{\pi} \int_{\left\{t \in\left[s_{1}, s_{2}\right]: x(t)<c\right\}} \frac{(x(t)-c) X(t, x(t))+y(t)^{2}}{\nu^{2}(x(t)-c)^{2}+y(t)^{2}} d t \\
& =\frac{\mu}{\pi} \int_{\left\{t \in\left[s_{1}, s_{2}\right]: x(t)>c\right\}} \frac{(x(t)-c) X(t, x(t))+y(t)^{2}}{\mu^{2}(x(t)-c)^{2}+y(t)^{2}} d t
\end{aligned}
$$

whenever

$$
\operatorname{rot}_{\psi}\left(s_{2}, s_{1} ; z\right) \in \mathbb{Z}
$$

From these relations, one can easily prove that when $\operatorname{rot}_{\psi}\left(s_{2}, s_{1} ; z\right) \in \mathbb{Z}$, then

$$
\operatorname{rot}_{\psi}\left(s_{2}, s_{1} ; z\right)=\frac{\mu \nu}{\pi(\mu+\nu)} \int_{s_{1}}^{s_{2}} \frac{(x(t)-c) X(t, x(t))+y(t)^{2}}{\psi^{2}(x(t))+y(t)^{2}} d t .
$$

Thus, in particular, we find that the integral

$$
\frac{\mu \nu}{\pi(\mu+\nu)} \int_{s_{1}}^{s_{2}} \frac{(x(t)-c) X(t, x(t))+y(t)^{2}}{\psi^{2}(x(t))+y(t)^{2}} d t
$$


is independent on the choice of $\mu, \nu>0$ when it is an integer (see [14], [42] for related results).

We are interested in finding $m T$-periodic solutions of (4) for some $m \in \mathbb{Z}_{0}^{+}$. Observe that from the $T$-periodicity of $X(\cdot, x)$, it follows that if $(x, y)$ is a $m T$ periodic solution of (4) for some $m \geq 2$, then the translated function $(x(\cdot-j T)$, $y(\cdot-j T))$ is a $m T$-periodic solution for each $1 \leq j \leq m-1$. We consider these solutions as belonging to the same periodicity class.

The following version of the generalized Poincaré - Birkhoff fixed point theorem is the main tool for the proof of our existence and multiplicity results.

Theorem 5. Let $\mathcal{A} \subset] a, b[\times \mathbb{R}$ be a closed annular region around $P=(c, 0)$ such that the inner and the outer boundaries, respectively $C_{1}$ and $C_{2}$, are simple closed curves. Suppose that $C_{1}$ is strictly star-shaped around $P$. For a fixed $m \in \mathbb{Z}_{0}^{+}$, assume

$$
z\left(t ; t_{0}, z_{0}\right) \neq P, \forall t_{0} \in\left[0, T\left[, \forall z_{0} \in C_{1}, \forall t \in\left[t_{0}, m T\right] .\right.\right.
$$

Let $\left.\psi_{i}:\right] A_{i}, B_{i}[\supset] a, b[, i=1,2$, be admissible functions with respect to $c$ such that

$$
\operatorname{rot}_{\psi_{1}}\left(m T ; z_{0}\right)>m_{1}+\delta_{\psi_{1}}, \forall z_{0} \in C_{1}
$$

and

$$
\operatorname{rot}_{\psi_{2}}\left(m T ; z_{0}\right)<m_{2}-\delta_{\psi_{2}}, \forall z_{0} \in C_{2}
$$

with $m_{1}, m_{2}$ in $\mathbb{Z}^{+}, m_{1} \geq m_{2}$. Then for every integer $k$ with $m_{2} \leq k \leq m_{1}$, equation

$$
x^{\prime \prime}+X(t, x)=0
$$

has at least two $m T$-periodic solutions not belonging to the same periodicity class, with $x(\cdot)-c$ having exactly $2 k$ zeros in the interval $[0, m T[$. Thus, in particular, equation (13) has at least $2\left(m_{1}-m_{2}+1\right)$ different $m T$-periodic solutions.

Remark that the conditions (11) and (12) are meaningful since from (10) taking $t_{0}=0$, the rotation numbers are defined. In our applications, (12) will be satisfied with $\delta_{\psi_{2}}=0$, by Remark 1 .

Proof. Fix an integer $k$ such that $m_{2} \leq k \leq m_{1}$. For $i=1,2$, let $D_{i}$ be the open region bounded by $C_{i}$. Consider, on $\bar{D}_{2}$, the function $\Psi$ defined by $\Psi\left(z_{0}\right)=z\left(m T ; z_{0}\right)$. By the assumptions concerning $X$ we know that $\Psi$ is a homeomorphism onto its image, moreover by the Liouville's theorem we have that $\Psi$ is area-preserving.

For each point $z_{0}=\left(x_{0}, y_{0}\right) \in \mathcal{S}, \Psi^{-1}\left(z_{0}\right)=z\left(-m T ; z_{0}\right)$. This is a consequence of the uniqueness of the solutions to the Cauchy problems and the $T$-periodicity in the first variable of $X$ from which we obtain $z\left(t ; z\left(-m T ; z_{0}\right)\right)=z\left(t-m T ; z_{0}\right)$ and the result follows.

We claim that

$$
\Psi^{-1}(P) \in D_{1} .
$$

Indeed, suppose by contradiction that $z_{*}=z(-m T ; P) \notin D_{1}$, that is $z\left(m T ; z_{*}\right)=$ $P$, with $z_{*} \notin D_{1}$. As $P \in D_{1}$, we can find $t_{1} \in\left[0, m T\left[\right.\right.$, such that $z_{1}:=z\left(t_{1} ; z_{*}\right) \in$ 
$C_{1}$. Fix now a non-negative integer $j$ such that $t_{0}:=t_{1}-j T \in[0, T[$ and consider the solution $z\left(\cdot ; t_{0}, z_{1}\right)$. By the uniqueness of the Cauchy problems and the $T$ periodicity of $X$ in the first variable we know that $z\left(t-j T ; t_{0}, z_{1}\right)=z\left(t ; t_{1}, z_{1}\right)$. Hence we obtain that for $t=m T-j T, z\left(t ; t_{0}, z_{1}\right)=z\left(m T ; t_{1}, z_{1}\right)=z\left(m T ; z_{*}\right)=P$ and observe that $t_{0}<t=m T-j T \leq m T$. This contradicts (10) and the claim is proved.

Consider the restriction of $\Psi$ to $\mathcal{A}$ that we still denote by $\Psi$. Arguing as above, we have that $\Psi(z) \neq P$ for each $z \in \mathcal{A}$. Hence we can consider a lifting of $\Psi$ to $E_{x-c}$. We choose the lifting $\tilde{\Psi}: \tilde{\mathcal{A}} \subset \mathbb{R}_{0}{ }^{+} \times \mathbb{R} \rightarrow \mathbb{R}_{0}{ }^{+} \times \mathbb{R}$ defined by $\tilde{\Psi}(r, \phi)=(f(r, \phi), \phi+g(r, \phi))$ where $f(r, \phi)=\left\|z\left(m T ; \Pi_{x-c}(r, \phi)\right)-P\right\|$ and $g(r, \phi)=$ $-2 \pi \operatorname{rot}\left(m T ; \Pi_{x-c}(r, \phi)\right)+2 k \pi$. By (11) we have that $\operatorname{rot}_{\psi_{1}}\left(m T ; \Pi_{x-c}(r, \phi)\right)>$ $m_{1}+\delta_{\psi_{1}}$, for each $(r, \phi) \in \Pi_{x-c}^{-1}\left(C_{1}\right)$, and so, applying Theorem 4 , we conclude that

$$
\operatorname{rot}\left(m T ; \Pi_{x-c}(r, \phi)\right)>m_{1}, \quad \text { for each }(r, \phi) \in \Pi_{x-c}^{-1}\left(C_{1}\right) .
$$

Arguing in the same manner, from (12) and Theorem 4, we have that

$$
\operatorname{rot}\left(m T ; \Pi_{x-c}(r, \phi)\right)<m_{2} \quad \text { for each }(r, \phi) \in \Pi_{x-c}^{-1}\left(C_{2}\right) .
$$

Thus we obtain $g(r, \phi)=-2 \pi \operatorname{rot}\left(m T ; \Pi_{x-c}(r, \phi)\right)+2 k \pi<0$ on $\Pi_{x-c}^{-1}\left(C_{1}\right)$ and $g(r, \phi)>0$ on $\Pi_{x-c}^{-1}\left(C_{2}\right)$.

We can apply now the W. Ding's generalized Poincaré - Birkhoff fixed point theorem, (see [10]), and obtain the existence of at least two geometrically distinct fixed points $\left(r_{k, i}, \phi_{k, i}\right)$, for $i=1,2$, of $\tilde{\Psi}$. For each $i=1,2, z_{k, i}:=\Pi_{x-c}\left(r_{k, i}, \phi_{k, i}\right)$ is a fixed point of $\Psi$ with $z_{k, i} \in \mathcal{A}$ and $z_{k, 1} \neq z_{k, 2}$. So, each of these points corresponds to a $m T$-periodic solution of (4). Arguing as in an observation in [35, p. 382], we can prove that these solutions do not belong to the same periodicity class. Moreover, since $g=0$ on the fixed points, we have $\operatorname{rot}\left(m T ; \Pi_{x-c}\left(r_{k, i}, \phi_{k, i}\right)\right)=k$. Hence, as all the solutions $(x(\cdot), y(\cdot))$ of $(4)$ with initial conditions on $\mathcal{A}$ satisfy $x^{\prime}(t) \neq 0$ for each $t$ such that $x(t)=c$, we conclude that for $i=1,2,\left(x_{k, i}(\cdot), y_{k, i}(\cdot)\right):=z\left(\cdot ; z_{k, i}\right)$ is such that $x_{k, i}(t)-c$ vanishes exactly $2 k$ times on $[0, m T[$. As $k$ is an arbitrary integer greater than or equal to $m_{2}$ and smaller than or equal to $m_{1}$ the result follows.

\section{Global extendability and some estimates}

In order to prove our main result we have to employ the global existence in the past and in the future of the solutions of a class of systems of the form of system (4). More precisely we are interested in systems of the form

$$
\left\{\begin{array}{l}
x^{\prime}=y \\
y^{\prime}=-f(x)+w(t, x)
\end{array}\right.
$$

where we suppose that $f:] a, b[\rightarrow \mathbb{R}$ is continuous and $w$ is continuous in $\mathbb{R} \times] a, b[$ and $T$-periodic in the first variable. Moreover the function $w$ will satisfy

$$
|w(t, x)| \leq l(t), \quad \forall t \in \mathbb{R}, x \in] a, b[,
$$

for some continuous and $T$-periodic function $l$ defined on $\mathbb{R}$. Clearly, we could assume $l$ to be constant, however we prefer not to use this condition at this moment 
in order to give a proof of the next result (Theorem 6 below) which is valid for a wider class of equations. In what follows we set

$$
l_{m}:=\int_{0}^{m T} l(t) d t
$$

i.e. the $L^{1}$-norm of $l(\cdot)$ in $[0, m T]$. We also denote by $F(x)$, for $\left.x \in\right] a, b[$, a primitive of $f(x)$.

We have the following general theorem concerning global existence of solutions of system (14).

Theorem 6. Assume (15) and suppose that there exists a constant $\mu \geq 0$ such that

$$
F(x)+\mu x^{2} \rightarrow+\infty \quad \text { for } x \rightarrow a^{+} \text {and for } x \rightarrow b^{-} .
$$

Then every noncontinuable solution $z(t)=(x(t), y(t))$ of (14) satisfying

$$
\left.x\left(t_{0}\right)=x_{0} \in\right] a, b\left[, \quad y\left(t_{0}\right)=y_{0}\right.
$$

is defined for all $t \in(-\infty,+\infty)$.

Clearly, the validity of Theorem 6 does not depend on the choice of the primitive F.

Proof. By (16) there exists a $\nu \geq 0$ such that $F(x)+\mu x^{2}+\nu \geq 0$ for every $\left.x \in\right] a, b[$. Define the function $V: \mathcal{S}=] a, b[\times \mathbb{R} \rightarrow \mathbb{R}$ by

$$
V(x, y)=F(x)+\left(\mu+\frac{1}{2}\right) x^{2}+\left(\nu+\frac{1}{2}\right)+\frac{1}{2} y^{2} .
$$

Claim: $\lim _{(x, y) \rightarrow \partial \mathcal{S}} V(x, y)=+\infty$.

In fact, if for some $k>0$ and some $(x, y), V(x, y) \leq k$, then we have $\frac{1}{2} x^{2}+\frac{1}{2} y^{2}+$ $\frac{1}{2} \leq k$ and $F(x)+\mu x^{2} \leq k$. Thus $|y| \leq \sqrt{2 k}$ and, by (16), there exists a compact interval $\left.I_{k} \subset\right] a, b\left[\right.$ such that $x \in I_{k}$. From this we conclude that $V(x, y)>k$ if $(x, y) \notin I_{k} \times[-\sqrt{2 k}, \sqrt{2 k}]$, and the claim follows.

Let us consider now a noncontinuable solution $z(\cdot)$ of (14)-(17). Suppose that $(x(t), y(t))$ is defined for $t \in] t_{-}, t_{+}\left[\right.$. Let us prove that $t_{+}=+\infty$, the proof that $t_{-}=-\infty$ is analogous.

Denote by $v:] t_{-}, t_{+}[\rightarrow \mathbb{R}$ the function defined by $v(t)=V(x(t), y(t))$. We have

$$
v^{\prime}(t)=2 \mu x(t) x^{\prime}(t)+x(t) x^{\prime}(t)+w(t, x(t)) x^{\prime}(t) .
$$

Hence $\forall t \in] t_{-}, t_{+}[$,

$$
\left|v^{\prime}(t)\right| \leq 2 \tilde{l}(t) v(t) \quad \text { with } \quad \tilde{l}(t)=\mu+\frac{1}{2}+\frac{|l(t)|}{2}
$$

from which we conclude

$$
v(t) \leq v\left(t_{0}\right) \exp \left(2\left|\int_{t_{0}}^{t} \tilde{l}(\tau) d \tau\right|\right) .
$$


If $t_{+}<+\infty$, then $(x(t), y(t)) \rightarrow \partial \mathcal{S}$ for $t \rightarrow t_{+}^{-}$and therefore by the previous claim $v(t)=V(x(t), y(t)) \rightarrow+\infty$ for $t \rightarrow t_{+}^{-}$, but this contradicts equation (18).

Remark 2. We can obtain the same result for a more general class of equations, indeed we can consider systems of the form

$$
\left\{\begin{array}{l}
x^{\prime}=y \\
y^{\prime}=-f(x)+w(t, x, y)
\end{array}\right.
$$

with $w$ satisfying the Carathéodory assumptions, and replace (15) by the more general condition

$$
|w(t, x, y)| \leq \alpha(t)+\beta(t)|x|+\gamma(t)|y|
$$

where $\alpha, \beta$ and $\gamma$ are nonnegative functions belonging to $L_{l o c}^{1}(\mathbb{R})$.

In the main case for our study in the following section (Theorem 7 ), we consider $a=-\infty$ and $F$ such that

$$
\lim _{x \rightarrow b^{-}} F(x)=+\infty
$$

In this situation, we can obtain global extendability of the solutions if $f$ is bounded from above in a neighbourhood of $-\infty$. Actually we have:

Corollary 5. Assume (15),(20) and

$$
\limsup _{x \rightarrow-\infty} f(x)<+\infty .
$$

Then every noncontinuable solution of system (14) is globally defined.

Proof. Let $M_{0}>0$ and $a_{0}<b$ be such that, according to (21), $f(x) \leq M_{0}$, for every $x \leq a_{0}$. Then $F(x) \geq F\left(a_{0}\right)-M_{0}\left|x-a_{0}\right|$, for every $x \leq a_{0}$ and so $\lim _{x \rightarrow-\infty} F(x)+$ $x^{2}=+\infty$. On the other hand, from (20) we have $\lim _{x \rightarrow b^{-}} F(x)+x^{2}=+\infty$. Thus equation (16) is satisfied for $\mu=1$ and the result follows via Theorem 6 .

If, instead of (21), we have

$$
\limsup _{x \rightarrow a^{+}} f(x)=+\infty
$$

with $a=-\infty$ or $a>-\infty$ and (20) holds, we can obtain global extendability of the solutions by studying a modified problem. More precisely, we fix a point $\left.a_{0} \in\right] a, b\left[\right.$, define $M=\sup \left\{f\left(a_{0}\right),|l|_{\infty}\right\}$ and denote by $k_{M}$ the greatest $x \leq a_{0}$ such that $f(x)=M+1$. By (22) such a $k_{M}$ always exists. Consider now the function $\hat{f}:]-\infty, b[\rightarrow \mathbb{R}$ defined by

$$
\hat{f}(x)= \begin{cases}f(x) & \text { if } x \geq k_{M}, \\ M+1 & \text { if } x<k_{M} .\end{cases}
$$

Observe that, by construction of $\hat{f}$ and from $f\left(a_{0}\right)<M+1$, we have $\hat{f}(x) \leq M+1$ for all $x \in\left(-\infty, a_{0}\right]$. On the other hand, if we take the primitive $\hat{F}(x)=\int_{a_{0}}^{x} \hat{f}(\xi) d \xi$ of $\hat{f}$, we have $\hat{F}(x)=F(x)-F\left(a_{0}\right)$, so that $\lim _{x \rightarrow b^{-}} \hat{F}(x)=+\infty$ follows from (20). 
Consider now the system

$$
\left\{\begin{array}{l}
x^{\prime}=y \\
y^{\prime}=-\hat{f}(x)+w(t, x) .
\end{array}\right.
$$

By Corollary 5 we know that every noncontinuable solution of (24)-(17), is defined on $\mathbb{R}$. The special choice of the truncation given in (23) guarantees that all of the periodic solutions we find for (24) are periodic solutions of (14) too. In fact we have

Lemma 7. If $(x(\cdot), y(\cdot))$ is a periodic solution of (24) then it is a periodic solution of (14), with the same period.

Proof. Let $x(\cdot): \mathbb{R} \rightarrow \mathbb{R}$ be the first component of a periodic solution of (24). If, by contradiction, $\min x=x\left(t^{\star}\right)<k_{M}$, for some $t^{\star} \in \mathbb{R}$, we obtain

$$
0 \leq x^{\prime \prime}\left(t^{\star}\right)=-\hat{f}\left(x\left(t^{\star}\right)\right)+w\left(t^{\star}, x\left(t^{\star}\right)\right) \leq-M-1+|l|_{\infty} \leq-1,
$$

and the result follows.

The same result holds on the Carathéodory setting with a slightly different proof.

Remark 3. In what follows we assume the uniqueness of solutions of the initial value problems (14)-(17) or (24)-(17). This will be accomplished in our applications by assuming $f$ of class $\mathcal{C}^{1}$ and $w$ locally lipschitzian in the $x$-variable. Observe that if the truncation leading to $\hat{f}$ is needed, then uniqueness is still guaranteed. Indeed, if $f$ is of class $\mathcal{C}^{1}$, then $\hat{f}$ has bounded slope at any point of its domain. In the sequel we denote by $z\left(\cdot ; t_{0}, z_{0}\right)$ the solution of (14)-(17) and set $z\left(\cdot ; z_{0}\right):=z\left(\cdot ; 0, z_{0}\right)$.

We proceed now with some technical lemmas which are useful for the construction of the inner boundary of the annulus to which we will apply the Poincaré Birkhoff fixed point theorem.

We choose a point $c$ in the domain of $f$ and consider, as a primitive of $f$, the function $F(x)=\int_{c}^{x} f(\xi) d \xi$. In the next result we restrict the study of the solutions of (14) to a strip $\left[a_{1}, b\left[\times \mathbb{R}\right.\right.$, where $a_{1}<c<b$ and $\left[a_{1}, b[\subset \operatorname{dom} f\right.$. We show that, under condition $(20)$, we can find a curve around $P=(c, 0)$ such that solutions starting from there do not leave the strip nor hit the point $P$, during the time interval $[0, m T]$, provided that $F\left(a_{1}\right)$ is large enough.

Lemma 8. Assume (15),(20), suppose $F(x)>0$ for $a_{1} \leq x<b, x \neq c$ and define $W(x, y)=\sqrt{2 F(x)+y^{2}}$, for $a_{1} \leq x<b$ and $y \in \mathbb{R}$. Let $m \in \mathbb{Z}_{0}^{+}$and $d>l_{m}$ be given. Suppose also that

$$
F\left(a_{1}\right) \geq 2 d^{2} .
$$

Let $\left.z_{0}=\left(x_{0}, y_{0}\right) \in\right] a_{1}, b\left[\times \mathbb{R}\right.$ be such that $W\left(x_{0}, y_{0}\right)=d$. Then, for any $t_{0} \in[0, T[$, the solution $z(t)=z\left(t ; t_{0}, z_{0}\right)$ is defined in $\left[t_{0}, m T\right]$ with $z(t) \neq P$ and $x(t)>a_{1}$ for every $t \in\left[t_{0}, m T\right]$. Moreover

$$
d-l_{m} \leq W(z(t)) \leq d+l_{m}, \quad \forall t \in\left[t_{0}, m T\right] .
$$

Proof. Consider the solution $z(t)$ defined on its maximal right interval of existence $\left[t_{0}, \tau[\right.$. Assume by contradiction, that there is a $\tilde{t} \in] t_{0}, \tau[$ with $\tilde{t} \leq m T$, such that 
$x(\tilde{t}) \leq a_{1}$ or $z(\tilde{t})=P$. Since the set $\left.\left.\mathcal{A}=\right]-\infty, a_{1}\right] \times \mathbb{R} \cup\{P\}$ is closed with $z_{0}=\left(x\left(t_{0}\right), y\left(t_{0}\right)\right) \notin \mathcal{A}$, there is a first time $\left.\left.t_{1} \in\right] t_{0}, \tilde{t}\right]$, such that $z\left(t_{1}\right) \in \mathcal{A}$ and $z(t) \notin \mathcal{A}, \quad \forall t_{0} \leq t<t_{1}$. Thus, in particular, $a_{1}<x(t)<b$ and $z(t) \neq P$, $\forall t_{0} \leq t<t_{1}$.

For $t_{0} \leq t<t_{1}$ we evaluate $\omega(t)=W(z(t))$, and have $\left|\omega^{\prime}(t)\right| \leq l(t)$, hence $\left|\omega(t)-\omega\left(t_{0}\right)\right| \leq l_{m}$, so that $\forall t \in\left[t_{0}, t_{1}[\right.$,

$$
d-l_{m} \leq W(z(t)) \leq d+l_{m}
$$

holds. Passing to the limit as $t \rightarrow t_{1}^{-}$we have

$$
0<d-l_{m} \leq W\left(z\left(t_{1}\right)\right) \leq d+l_{m}<2 d .
$$

By the choice of $t_{1}$ we have $x\left(t_{1}\right)=a_{1}$ or $z\left(t_{1}\right)=P=(c, 0)$. Now, if $x\left(t_{1}\right)=a_{1}$ we find

$$
\sqrt{2 F\left(a_{1}\right)} \leq \sqrt{2 F\left(a_{1}\right)+y\left(t_{1}\right)^{2}}<2 d
$$

and therefore,

$$
F\left(a_{1}\right)<2 d^{2},
$$

a contradiction to equation (25). On the other hand, if $z\left(t_{1}\right)=(c, 0)$, we have

$$
0<d-l_{m} \leq W\left(z\left(t_{1}\right)\right)=W(c, 0)=0
$$

a contradiction.

Thus we have seen that, either there is no $\tilde{t}$ as above or, if any of such $\tilde{t}$ exists, then it must be $\tilde{t}>m T$. In the latter case we are done. In the former case, we conclude that $z(t) \in] a_{1}, b\left[\times \mathbb{R}\right.$ and (27) holds for all $t \in\left[t_{0}, \tau[\right.$. Therefore, we have that $z(t)$ belongs to the set $W^{-1}([0,2 d]$ ) (which is compact by $(20)$ ) for all the forward time where it is defined. Hence $\tau=+\infty$ and also $z(t) \neq P$ and $x(t)>a_{1}$, for every $t \in\left[t_{0},+\infty[\right.$.

Thus, in any case, we conclude that $z(t) \neq P$ and $x(t)>a_{1}$ for every $t \in\left[t_{0}, m T\right]$ and equation (26) follows now, as (27), from the fact that $\left|\omega^{\prime}(t)\right| \leq l(t)$ if $z(t) \in$ $\left[a_{1}, b[\times \mathbb{R} \backslash\{P\}\right.$.

The next lemma describes the geometry of the level sets of $W$ in the strip $\left[a_{1}, b[\times \mathbb{R}\right.$.

Lemma 9. Under the conditions of Lemma 8, suppose that $f(c)=0$ and $f(x)(x-c)$ $>0$, for each $x \in\left[a_{1}, b\left[, x \neq c\right.\right.$. Then $C_{1}=\left\{(x, y) \in\left[a_{1}, b[\times \mathbb{R}: W(x, y)=d\}\right.\right.$ is a simple closed curve strictly star-shaped around $P=(c, 0)$ contained in the strip $\left.\mathcal{S}_{1}=\right] a_{1}, b[\times \mathbb{R}$.

Proof. As $W(P)=0, W\left(a_{1}, y\right) \geq 2 d$ for each $y \in \mathbb{R}$ and $\lim _{x \rightarrow b^{-}} W(x, y)=$ $+\infty$, uniformly with respect to $y$, there exists a compact interval, $\left[a^{*}, b^{*}\right]$ with $\left.c \in\left[a^{*}, b^{*}\right] \subset\right] a_{1}, b\left[\right.$ such that for each $(x, y) \in(] a_{1}, b\left[\backslash\left[a^{*}, b^{*}\right]\right) \times \mathbb{R}$ we have $W(x, y)>$ $\frac{3}{2} d$. Moreover $W(x, y) \geq|y|$ and so we have $W(x, y)>\frac{3}{2} d$ for every $(x, y) \in$ $\mathcal{S}_{1} \backslash\left(\left[a^{*}, b^{*}\right] \times[-3 d / 2,3 d / 2]\right)$. Let $\theta \in[0,2 \pi]$ be fixed and consider the open interval ] $0, R_{\theta}\left[=\left\{r>0: P+(r \cos \theta, r \sin \theta) \in \mathcal{S}_{1}\right\}\right.$, with $R_{\theta} \leq+\infty$.

We claim that for each $\theta \in[0,2 \pi]$, there exists a unique $\left.r_{\theta} \in\right] 0, R_{\theta}[$ such that $P+\left(r_{\theta} \cos \theta, r_{\theta} \sin \theta\right) \in C_{1}$. Indeed let us consider for any $\theta \in[0,2 \pi]$ the function 
$\left.\xi_{\theta}:\right] 0, R_{\theta}\left[\rightarrow \mathbb{R}\right.$ given by $\xi_{\theta}(r)=W(P+(r \cos \theta, r \sin \theta))$. Notice that $\xi_{2 \pi} \equiv \xi_{0}$. By direct computation we have $\xi_{\theta}^{\prime}(r)>0$ for each $r>0$. By what we proved above we conclude that $\lim _{r \rightarrow R_{\theta}^{-}} \xi_{\theta}(r)>d$ and, as $\lim _{r \rightarrow 0^{+}} \xi_{\theta}(r)=0$, the result follows.

By the claim, $C_{1}=\left\{P+\left(r_{\theta} \cos \theta, r_{\theta} \sin \theta\right): \theta \in[0,2 \pi]\right\}$, and therefore $C_{1}$ is a simple closed curve strictly star-shaped around $P$ and contained in $\mathcal{S}_{1}$.

In the application of these results to equation $(E)_{s}$ the function $f(x)$ will play the role of $g(x)-s$ and the point $c$ in Lemma 8 and Lemma 9 will be such that $g(c)=s$. Precisely, for $g:] a, b\left[\rightarrow \mathbb{R}\right.$ and $G\left(b^{-}\right)=+\infty$, with $G$ a primitive of $g$, we will fix a point $a_{1}$ with $a<a_{1}<b$ and after this we will choose $\left.c \in\right] a_{1}, b$ [ with $g(c)=s$. Then we will have that the point $c$ moves toward $b$ as $s \rightarrow+\infty$. For the validity of the conditions on $F$ in Lemmas 8 and 9 , we will require $g$ strictly increasing in a neighbourhood of $b$.

We conclude the section with a consequence of the global extendability of the solutions (cf. [18, Proposition 3.2]) whose proof is standard:

Lemma 10. Under the conditions (15) and (16), or any other set of conditions ensuring the global continuability, we have that for every compact $\mathcal{K} \subset \mathcal{S}=] a, b[\times \mathbb{R}$ and each $m \in \mathbb{Z}_{0}^{+}$there exists a compact $\mathcal{M}=\mathcal{M}(\mathcal{K}, m) \subset \mathcal{S}$ such that if $z_{0} \notin \mathcal{M}$ then $z\left(t ; z_{0}\right) \notin \mathcal{K}, \forall t \in[-m T, m T]$.

\section{MAIN RESUlts}

Let $g:] a, b[\rightarrow \mathbb{R}$ be continuously differentiable and let $w=w(t, x): \mathbb{R} \times] a, b[\rightarrow \mathbb{R}$ be continuous, $T$-periodic in the $t$-variable and locally lipschitzian in the $x$-variable. Note that we can reduce ourselves to this situation also when $g$ is locally lipschitzian in $] a, b\left[\right.$ and of class $\mathcal{C}^{1}$ only in a neighbourhood of $a$ and $b$. Indeed this is just a matter of discarding a bounded lipschitz term on $w$. Let $G$ be a primitive of $g$ on ]$a, b[$ and suppose that, as in Section 3,

$$
|w(t, x)| \leq l(t), \quad \forall t \in \mathbb{R}, x \in] a, b[
$$

for some continuous and $T$-periodic function $l$ defined on $\mathbb{R}$. Recall also that $l_{m}=$ $\int_{0}^{m T} l(t) d t=m l_{1}$.

Using the truncation argument previously described, it is possible to see that if $\lim \sup _{x \rightarrow a^{+}} g(x)=+\infty$, then we can reduce the problem of the search of periodic solutions for $(E)_{s}$ to an equivalent problem with $g$ defined in $(-\infty, b[$ and such that $\lim \sup _{x \rightarrow-\infty} g(x)<+\infty$. By virtue of this remark which is explained with more details in the proof of Theorem 8 , we suppose at the beginning that

$$
] a, b[=(-\infty, b[.
$$

Now we are in position to state and prove our first main result. To this end we introduce some basic assumptions for the nonlinear function $g$, which are:

$$
\limsup _{x \rightarrow-\infty} g(x)<+\infty \text { and } \limsup _{x \rightarrow-\infty} g^{\prime}(x) \leq \alpha<+\infty,
$$

with $\alpha \geq 0$,

$$
0<\gamma \leq \liminf _{x \rightarrow b^{-}} g^{\prime}(x) \leq \limsup _{x \rightarrow b^{-}} g^{\prime}(x) \leq \beta \leq+\infty .
$$

Recall also that $\lambda_{i}=\left(\frac{2 \pi i}{T}\right)^{2}$, for $i \in \mathbb{Z}_{0}^{+}$, is the $i$-th positive eigenvalue of the operator $x \mapsto-x^{\prime \prime}$ with the $T$-periodic boundary conditions. 
Theorem 7. Assume $(28),(29)$. Suppose that $\lim _{x \rightarrow b^{-}} G(x)=+\infty$, where $G$ is a primitive of $g$, and let $k \in \mathbb{Z}_{0}^{+}$be such that $\left(\frac{2 \sqrt{\alpha} \sqrt{\beta}}{\sqrt{\alpha}+\sqrt{\beta}}\right)^{2}<\lambda_{k}$ (when $\beta=+\infty, 4 \alpha<$ $\left.\lambda_{k}\right)$. Then we have:

- If $\beta<+\infty$ and $\lambda_{j}<\gamma$, for some integer $j \geq k$, then equation $(E)_{s}$ has at least $2(j-k+1)$ harmonic solutions for each sufficiently large positive $s$. More precisely, there is $s\left(l_{1}\right) \in \mathbb{R}^{+}$such that for each $s>s\left(l_{1}\right)$ and for each integer $r$, with $k \leq r \leq j$, there exist two $T$-periodic solutions $x_{i}(\cdot), i=1,2$, of $(E)_{s}$, such that for each $i, x_{i}(\cdot)-g^{-1}(s)$ has exactly $2 r$ zeros in the interval $[0, T[$.

- If $\gamma=+\infty$, then for each integer $j \geq k$ equation $(E)_{s}$ has at least $2(j-k+1)$ harmonic solutions for each sufficiently large positive s. More precisely, there is $s\left(j, l_{1}\right) \in \mathbb{R}^{+}$such that for each $s>s\left(j, l_{1}\right)$ and for each integer $r$, with $k \leq r \leq j$, there exist two T-periodic solutions $x_{i}(\cdot), i=1,2$, of $(E)_{s}$, such that for each $i, x_{i}(\cdot)-g^{-1}(s)$ vanishes exactly $2 r$ times in the interval $[0, T[$.

The meaning of $g^{-1}(s)$ is the following: choose $0<\gamma_{1}<\gamma$ and note that by (29), $g^{\prime}(x) \geq \gamma_{1}>0$ for $x$ in a left neighbourhood of $b$ and this fact, together with $G\left(b^{-}\right)=+\infty$ in the case $b<+\infty$, implies that $g\left(b^{-}\right)=+\infty$. Hence for every $s>0$ sufficiently large there is a unique $c_{s}$ such that $g\left(c_{s}\right)=s$, with $c_{s}$ in a left neighbourhood of $b$. We set $g^{-1}(s):=c_{s}$. This conventional notation will be used throughout.

In order to demonstrate Theorem 7 we are going to state some lemmas whose proofs are postponed to the next section. These lemmas concern solutions of the planar equation

$$
\left\{\begin{array}{l}
x^{\prime}=y \\
y^{\prime}=-g(x)+s+w(t, x) .
\end{array}\right.
$$

More precisely we estimate the rotation numbers, with respect to some systems of coordinates, of the solutions of $(\mathcal{E})_{s}$ whose initial conditions lie on some curves that we construct. The curves are different in the cases $\gamma=+\infty$ and $\beta<+\infty$, but for simplicity and as no confusion arises, we use the same notations for these two different cases. In order to perform this program we assume the uniqueness of the solutions for the Cauchy problems associated to $(\mathcal{E})_{s}$. Observe that the uniqueness is guaranteed by the regularity conditions on $g$ and $w$. As to the global existence, this is not required in Lemma 11 below for which we employ Lemma 8. On the other hand, global existence is guaranteed in next Lemma 12 where we use the condition $G\left(b^{-}\right)=+\infty$ and $\gamma>0$. Indeed, these two assumptions imply that $F\left(b^{-}\right)=+\infty$, for $f(x)=g(x)-s$ and $F(x)$ any primitive of $f(x)$ on the interval $(-\infty, b[$. Moreover, $g(x)-s$ is bounded from above on a neighbourhood of $-\infty$, by the first assumption in (28), and thus Corollary 5 applies ensuring the global extendability of the solutions.

Lemma 11. Assume (29) and suppose that $\lim _{x \rightarrow b^{-}} G(x)=+\infty$ where $G$ is a primitive of $g$.

- If $\beta<+\infty$ and $\lambda_{j}<\gamma$, for some $j \in \mathbb{Z}^{+}$, then there are constants $a_{1}<b$ and $s\left(l_{1}\right)>0$ such that for each $s>s\left(l_{1}\right)$ there exist a strictly star-shaped curve $\left.\mathcal{C}_{1}^{s} \subset\right] a_{1}, b\left[\times \mathbb{R}\right.$ around $P_{s}=\left(g^{-1}(s), 0\right)$, and a function $\psi_{1}: \mathbb{R} \rightarrow \mathbb{R}$ admissible with respect to $g^{-1}(s)$, with $\delta_{\psi_{1}}=0$, satisfying: 
i) $\forall t_{0} \in\left[0, T\left[, \forall z_{0} \in \mathcal{C}_{1}^{s}: z\left(t ; t_{0}, z_{0}\right) \neq P_{s}\right.\right.$ and $x(t)>a_{1}, \forall t \in\left[t_{0}, T\right]$,

ii) $\operatorname{rot}_{\psi_{1}}\left(T ; z_{0}\right)>j, \forall z_{0} \in \mathcal{C}_{1}^{s}$.

- If $\gamma=+\infty$, for every $j \in \mathbb{Z}^{+}$, there are constants $a_{1}^{j}<b$ and $s\left(j, l_{1}\right)>0$ such that for each $s>s\left(j, l_{1}\right)$ there exist a strictly star-shaped curve $\left.\mathcal{C}_{1}^{s} \subset\right] a_{1}^{j}, b[\times \mathbb{R}$ around $P_{s}=\left(g^{-1}(s), 0\right)$, and a function $\psi_{2}: \mathbb{R} \rightarrow \mathbb{R}$, admissible with respect to $g^{-1}(s)$, satisfying:

i) $\forall t_{0} \in\left[0, T\left[, \forall z_{0} \in \mathcal{C}_{1}^{s}: z\left(t ; t_{0}, z_{0}\right) \neq P_{s}\right.\right.$ and $x(t)>a_{1}^{j}, \forall t \in\left[t_{0}, T\right]$,

ii) $\operatorname{rot}_{\psi_{2}}\left(T ; z_{0}\right)>j+1 / 4, \forall z_{0} \in \mathcal{C}_{1}^{s}$

Lemma 12. Under the assumptions of Theorem 7, for each $s$ such that $\mathcal{C}_{1}^{s}$ is defined according to the previous lemma, there exist a simple closed curve $\mathcal{C}_{2}^{s}$, with $\mathcal{C}_{1}^{s} \subset$ int $\mathcal{C}_{2}^{s}$, and an admissible function $\psi$ with $\delta_{\psi}=0$, such that for every $z(\cdot)$ solution of system $(\mathcal{E})_{s}$ with initial point $z_{0} \in \mathcal{C}_{2}^{s}$, we have $\operatorname{rot}_{\psi}\left(T ; z_{0}\right)<k$.

Proof of Theorem 7. Consider at first the case when $\beta<+\infty$. By Lemma 11, for each $s>s\left(l_{1}\right)$, there exists a strictly star-shaped curve $\mathcal{C}_{1}^{s}$ around $P_{s}=\left(g^{-1}(s), 0\right)$ such that for each $t_{0} \in\left[0, T\left[\right.\right.$ and $z_{0} \in \mathcal{C}_{1}^{s}, z\left(t ; t_{0}, z_{0}\right) \neq P_{s}, \forall t \in\left[t_{0}, T\right]$. Moreover, by Lemma 11, there is an admissible function $\psi_{1}$ such that for every $z_{0} \in \mathcal{C}_{1}^{s}$ we have $\operatorname{rot}_{\psi_{1}}\left(T ; z_{0}\right)>j=j+\delta_{\psi_{1}}$. Let $s>s\left(l_{1}\right)$ be fixed and apply Lemma 12. There exist a simple closed curve $\mathcal{C}_{2}^{s}$ surrounding $\mathcal{C}_{1}^{s}$, i.e. $\mathcal{C}_{1}^{s} \subset$ int $\mathcal{C}_{2}^{s}$ and an admissible function $\psi$ such that for every $z_{0} \in \mathcal{C}_{2}^{s}$ we have $\operatorname{rot}_{\psi}\left(T ; z_{0}\right)<k=k-\delta_{\psi}$.

Then we can apply Theorem 5 , where $\mathcal{A}$ is the annular region bounded by $\mathcal{C}_{1}^{s}$ and $\mathcal{C}_{2}^{s}$ and $\left.X: \mathbb{R} \times\right]-\infty, b[\rightarrow \mathbb{R}$ is given by $X(t, x)=g(x)-s-w(t, x)$, and the result follows.

Secondly, consider the case $\gamma=+\infty$. The argument is completely similar to the previous one with respect to the property $z\left(t ; t_{0}, z_{0}\right) \neq P_{s}$ for $t \in\left[t_{0}, T[\right.$ and the rotation estimate for $\psi$ on the curve $\mathcal{C}_{2}^{s}$. As to the rotation on $\mathcal{C}_{1}^{s}$, Lemma 11 now ensures the existence of an admissible function $\psi_{2}$ such that for every $z_{0} \in \mathcal{C}_{1}^{s}$ we have $\operatorname{rot}_{\psi_{2}}\left(T ; z_{0}\right)>j+(1 / 4)$ when $s>s\left(j, l_{1}\right)$. Recalling Theorem 4 we conclude that $\operatorname{rot}_{\psi_{2}}\left(T ; z_{0}\right)>j+\delta_{\psi_{2}}$ for $z_{0} \in \mathcal{C}_{1}^{s}$. Then the conclusion follows as above by Theorem 5 .

The next result is a variant of Theorem 7 for the case in which condition (28) is not satisfied. Moreover, we don't require $a=-\infty$ anymore. Then we have:

Theorem 8. Assume $-\infty \leq a<b \leq+\infty$ and suppose

$$
\limsup _{x \rightarrow a^{+}} g(x)=+\infty .
$$

If we further assume (29) and $\lim _{x \rightarrow b^{-}} G(x)=+\infty$ where $G$ is a primitive of $g$, then all the conclusions of Theorem 7 hold with $k=1$.

Proof. Suppose at first $\beta<+\infty$ and that $\lambda_{j}<\gamma$ for some integer $j \geq 1$. Since (29) is assumed and $\lim _{x \rightarrow b^{-}} G(x)=+\infty$, we can apply Lemma 11 and derive the existence of some constants $a_{1}$, with $a<a_{1}<b$, and $s\left(l_{1}\right)>0$, such that for each $s>s\left(l_{1}\right)$ there exist a strictly star-shaped curve $\mathcal{C}_{1}^{s}$ around $P_{s}=\left(g^{-1}(s), 0\right)$, with $\left.\mathcal{C}_{1}^{s} \subset\right] a_{1}, b\left[\times \mathbb{R}\right.$ and a function $\psi_{1}: \mathbb{R} \rightarrow \mathbb{R}$ admissible with respect to $g^{-1}(s)$ with $\delta_{\psi_{1}}=0$, satisfying i) and ii) of the Lemma 11. Fix $s>s\left(l_{1}\right)$, let $c=g^{-1}(s)$

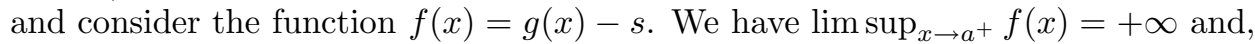
as $\gamma>0$ and $\lim _{x \rightarrow b^{-}} G(x)=+\infty, \lim _{x \rightarrow b^{-}} F(x)=+\infty$ holds where $F(x)=$ 
$\int_{c}^{x} f(\xi) d \xi$. Accordingly, we can apply the truncation argument exposed on Section 3 and obtain a function $\hat{f}:]-\infty, b\left[\rightarrow \mathbb{R}\right.$ defined as in (23) where $k_{M}$ is the greatest $x$ with $a<x<a_{1}$, such that $f(x)=\sup \left\{f\left(a_{1}\right),|l|_{\infty}\right\}+1$. Moreover we know that solutions of system (24) are globally defined and periodic solutions of this modified problem are periodic solutions of system $(\mathcal{E})_{s}$.

Let us now study the modified problem (24) which can be written in the form

$$
\left\{\begin{array}{l}
x^{\prime}=y \\
y^{\prime}=-\hat{g}(x)+s+w(t, x)
\end{array}\right.
$$

with $\hat{g}(x)=\hat{f}(x)+s$. First of all notice that $\hat{g}=g$ for $x \geq a_{1}$ and recall that all the preceding conclusions of Lemma 11 remain valid for system (31). On the other hand $\hat{g}$ satisfies all the hypothesis of Theorem 7 if we replace $\alpha$ by zero and take $k=1$. Then we apply Lemma 12 to system (31) and conclude with the existence of a simple closed curve $\mathcal{C}_{2}^{s}$ with $\mathcal{C}_{1}^{s} \subset \operatorname{int} \mathcal{C}_{2}^{s}$ and an admissible function $\psi$ with $\delta_{\psi}=0$, such that for every $z(\cdot)$ solution of system (31) with initial condition $z_{0} \in \mathcal{C}_{2}^{s}$ we have $\operatorname{rot}_{\psi}\left(T ; z_{0}\right)<1$. The result follows now from Theorem 5 taking into account that, as we previously remarked, periodic solutions of the modified problem are periodic solutions of $(\mathcal{E})_{s}$.

For the case $\gamma=+\infty$ the argument is similar to the one just given and therefore it is omitted (see also the proof of the main theorem).

\section{Proofs of the Auxiliary lemmas}

Proof of Lemma 11. First of all notice that this lemma is valid in both of the cases $a=-\infty$ and $a>-\infty$. Indeed, all of the reasoning we make on this proof concerns the behaviour of the function $g$ and the solutions of system $(\mathcal{E})_{s}$ on a left neighbourhood of $b$.

For each $c \in] a, b\left[\right.$ define the function $\left.f_{c}:\right] a, b\left[\rightarrow \mathbb{R}\right.$ by $f_{c}(x)=g(x)-g(c)$. Clearly $f_{c}(c)=0$, for every $c$.

- Suppose $\beta<+\infty$ in (29). In this case, as $\lim _{x \rightarrow b^{-}} G(x)=+\infty$, we have $b=+\infty$. Choose a sufficiently small constant $\varepsilon>0$. A posteriori considerations will show that a suitable choice for $\varepsilon$ is $\varepsilon=\frac{\pi}{T}\left(\frac{\sqrt{\gamma} T}{2 \pi}-j\right)$, where the positive integer $j$ is such that $\lambda_{j}<\gamma$. We also fix two constants $h$ and $M$ with $\sqrt{\gamma}>h>\sqrt{\gamma}-\varepsilon>0$ and $M=\sqrt{\beta+1}$. By assumption (29), there exists an $a_{1}$ with $a<a_{1}<+\infty$ such that

$$
0<h^{2} \leq g^{\prime}(x) \leq M^{2}, \quad \text { for } x \geq a_{1} .
$$

Take $d$ such that $d>l_{1}+\frac{M l_{1}}{\varepsilon h T}$ and choose a constant $c_{j}>a_{1}+\frac{2 d}{h}$. Thus we can define $s\left(l_{1}\right):=g\left(c_{j}\right)$.

Note that $g:\left[c_{j},+\infty\right) \rightarrow\left[s\left(l_{1}\right),+\infty\right)$ is strictly increasing and onto, thus for any $s>s\left(l_{1}\right)$ there is a unique $c>c_{j}$ with $g(c)=s$. In what follows, for any fixed $c>c_{j}$, we write $c=g^{-1}(s), g(c)=s$, and set $f:=f_{c}$. With these positions, a solution of (14)-(17) will be a solution of $(\mathcal{E})_{s^{-}}(17)$.

With the notations in Section 3, as we have $F(x)=\int_{c}^{x} f(\xi) d \xi>0$ for $x \neq c$ and $x \geq a_{1}$, we can consider the level lines of the function $W(x, y)$. We will prove that the curve

$$
\mathcal{C}_{1}^{s}:=\left\{( x , y ) \in \left[a_{1}, b[\times \mathbb{R}: W(x, y)=d\}\right.\right.
$$


and the function $\left.\psi_{1}:\right]-\infty,+\infty\left[\rightarrow \mathbb{R}\right.$ defined by $\psi_{1}(x)=(x-c) h$, will satisfy i) and ii) of the lemma. Notice that $\psi_{1}$ is admissible with respect to $c$ and that $\delta_{\psi_{1}}=0$ by Remark 1 in Section 2 .

Since $\liminf \inf _{x \rightarrow b^{-}} g^{\prime}(x) \geq \gamma>0$ and $b=+\infty$, we have that $F(+\infty)=+\infty$, so that (20) is satisfied. Moreover, as $c>c_{j}$,

$$
F\left(a_{1}\right) \geq \frac{\left(c-a_{1}\right)^{2} h^{2}}{2}>2 d^{2} .
$$

Hence we can apply Lemma 9 and Lemma 8 in order to conclude that $\mathcal{C}_{1}^{s}$ is a strictly star-shaped curve around $\left(g^{-1}(s), 0\right)$ and, for each $t_{0} \in[0, T[$ and each $z_{0}=\left(x_{0}, y_{0}\right) \in \mathcal{C}_{1}^{s}$, the corresponding solution $z(\cdot)$ of $(14)-(17)$ is defined on $\left[t_{0}, T\right]$ and satisfies $z(t) \neq(c, 0)$ and $x(t)>a_{1}$ for each $t \in\left[t_{0}, T\right]$. Therefore i) follows.

To prove ii) we preliminarly observe that

$$
f(x)(x-c)=(f(x)-f(c))(x-c) \geq h^{2}(x-c)^{2},
$$

and

$$
W(x, y)^{2}=2 F(x)+y^{2} \leq M^{2}(x-c)^{2}+y^{2} \leq(M / h)^{2}\left(h^{2}(x-c)^{2}+y^{2}\right)
$$

hold for every $x \geq a_{1}$.

Let us consider now the coordinate system corresponding to $\psi_{1}$ defined in Section 2. We obtain the following expression for the rotation number according to (6):

$$
\begin{aligned}
\operatorname{rot}_{\psi_{1}}(T ; z) & =\frac{1}{2 \pi} \int_{0}^{T} \frac{h\left[y(t)^{2}+f(x(t))(x(t)-c)-w(t, x(t))(x(t)-c)\right]}{h^{2}(x(t)-c)^{2}+y(t)^{2}} d t \\
& \geq \frac{h T}{2 \pi}-\frac{1}{2 \pi} \int_{0}^{T} \frac{l(t)}{\left(h^{2}(x(t)-c)^{2}+y(t)^{2}\right)^{1 / 2}} d t .
\end{aligned}
$$

As, by Lemma 8, solutions $z(\cdot)$ of (14)-(17) with $t_{0}=0$ and $z_{0}=\left(x_{0}, y_{0}\right) \in \mathcal{C}_{1}^{s}$ satisfy $x(t)>a_{1}$ and $W(z(t)) \geq d-l_{1}>0$ for each $t \in[0, T]$, we obtain

$$
\operatorname{rot}_{\psi_{1}}\left(T ; z_{0}\right) \geq \frac{h T}{2 \pi}-\frac{M l_{1}}{2 \pi h\left(d-l_{1}\right)}
$$

and finally, by the choice of $\varepsilon, h$ and $d$ we find that $\operatorname{rot}_{\psi_{1}}\left(T ; z_{0}\right)>j$, thus proving ii).

- Suppose $\gamma=+\infty$ in (29). Let us fix a nonnegative integer $j$. Choose $M>$ $\frac{2 \pi}{T}\left(j+\frac{1}{4}\right)$. By $(29)$, there exists an $\left.a_{1}^{j} \in\right] a, b[$ such that

$$
g^{\prime}(x)>M^{2} \quad \text { for } a_{1}^{j} \leq x<b .
$$

Hence, using the fact that $\lim _{x \rightarrow b^{-}} G(x)=+\infty$, we have that the function $g$ : $\left[a_{1}^{j}, b\left[\rightarrow\left[g\left(a_{1}^{j}\right),+\infty\right)\right.\right.$ is strictly increasing and onto, thus, for any $s>g\left(a_{1}^{j}\right)$ there is a unique $c \in] a_{1}^{j}, b\left[\right.$ with $g(c)=s$. As before, we write $c=g^{-1}(s)$. Choose a constant $d>l_{1}+\frac{l_{1}}{2 \pi}\left(\frac{M T}{2 \pi}-j-\frac{1}{4}\right)^{-1}$. 
We claim that there exists $\left.c_{j} \in\right] a_{1}^{j}, b\left[\right.$ such that for every $c>c_{j}$, we have

$$
F_{c}\left(a_{1}^{j}\right)>2 d^{2}
$$

where $F_{c}(x)=\int_{c}^{x} f_{c}(\xi) d \xi$. Indeed, integrating by parts, we find

$$
F_{c}\left(a_{1}^{j}\right)=\int_{c}^{a_{1}^{j}}(g(\xi)-g(c)) d \xi=\int_{a_{1}^{j}}^{c}\left(\xi-a_{1}^{j}\right) g^{\prime}(\xi) d \xi .
$$

Then, by a change of variable and using the monotonicity of $g^{-1}$, we have

$$
\begin{aligned}
\int_{a_{1}^{j}}^{c}\left(\xi-a_{1}^{j}\right) g^{\prime}(\xi) d \xi & =\int_{g\left(a_{1}^{j}\right)}^{g(c)}\left(g^{-1}(\eta)-a_{1}^{j}\right) d \eta \\
& \geq \int_{g\left(a_{1}^{j}\right)}^{g\left(\varepsilon+a_{1}^{j}\right)}\left(g^{-1}(\eta)-a_{1}^{j}\right) d \eta+\varepsilon\left(g(c)-g\left(\varepsilon+a_{1}^{j}\right)\right),
\end{aligned}
$$

where $\varepsilon>0$ is a fixed sufficiently small constant. Using $\lim _{x \rightarrow b^{-}} g(x)=+\infty$, we can conclude the proof of the claim taking $c_{j}$ sufficiently near to $b^{-}$.

At this moment, we define $s\left(j, l_{1}\right):=g\left(c_{j}\right)$ and proceed with our argument choosing an arbitrary but fixed $c>c_{j}$ and setting $f:=f_{c}$. With these positions, a solution of (14)-(17) will be a solution of $(\mathcal{E})_{s^{-}}(17)$ with $s=g(c)$.

With the notations in Section 3, as we have $F(x)=\int_{c}^{x} f(\xi) d \xi>0$ for $x \neq$ $c$ and $x \in\left[a_{1}^{j}, b[\right.$, we can consider the level lines of the function $W(x, y)$. Since $\liminf _{x \rightarrow b^{-}} g^{\prime}(x) \geq \gamma>0$ and $\lim _{x \rightarrow b^{-}} G(x)=+\infty$, we have that $F\left(b^{-}\right)=+\infty$, so that equation (20) is satisfied in both cases $b<+\infty$ or $b=+\infty$. Since we have already verified (25) (see (32)), we are in position to apply Lemma 9 and Lemma 8. Consequently, for

$$
\mathcal{C}_{1}^{s}=\left\{( x , y ) \in \left[a_{1}^{j}, b[\times \mathbb{R}: W(x, y)=d\},\right.\right.
$$

we have, by Lemma 9 , that $\mathcal{C}_{1}^{s}$ is a strictly star-shaped curve around the point $P_{s}=\left(g^{-1}(s), 0\right)=(c, 0)$. On the other hand, by Lemma 8, solutions of the Cauchy problem (14)-(17) with initial conditions $t_{0} \in\left[0, T\left[\right.\right.$ and $z_{0}=\left(x_{0}, y_{0}\right) \in \mathcal{C}_{1}^{s}$ are defined on $\left[t_{0}, T\right]$ and satisfy $z(t) \neq P_{s}$ and $x(t)>a_{1}^{j}$ for each $t \in\left[t_{0}, T\right]$. Therefore i) follows.

To prove ii) we preliminarly observe that applying Lagrange's theorem, and recalling that $f^{\prime}(x)>M^{2}$ for $x \geq a_{1}^{j}$, the following estimate

$$
\frac{f(x)^{2}}{2 F(x)}=\frac{(f(x)-f(c))^{2}}{2(F(x)-F(c))}>M^{2}
$$

holds for all $x \in\left[a_{1}^{j}, b\left[\right.\right.$, where the function $x \rightarrow \frac{f(x)^{2}}{2 F(x)}$ is extended by continuity at $x=c$, assuming the value $f^{\prime}(c)$.

Let $\left.\psi_{2}:\right]-\infty, b[\rightarrow \mathbb{R}$ be an admissible function with respect to $c$ such that $\psi_{2}(x)=\sqrt{2 F(x)} \operatorname{sgn}(x-c)$ for all $x$ with $a_{1}^{j} \leq x<b$ and consider, as in Section 2 , the coordinate system corresponding to $\psi_{2}$. Using the fact that solutions $z(\cdot)$ of 
(14)-(17) with $t_{0}=0$ and $z_{0}=\left(x_{0}, y_{0}\right) \in \mathcal{C}_{1}^{s}$ verify $x(t)>a_{1}^{j}$ for each $t \in[0, T]$, we obtain the following formula for the rotation number according to (6):

$$
\begin{aligned}
\operatorname{rot}_{\psi_{2}}(T ; z) & =\frac{1}{2 \pi} \int_{0}^{T}\left(\frac{|f(x(t))|}{\sqrt{2 F(x(t))}}-\frac{w(t, x(t)) \sqrt{2 F(x(t))} \operatorname{sgn}(x(t)-c)}{2 F(x(t))+y^{2}(t)}\right) d t \\
& >\frac{M T}{2 \pi}-\frac{1}{2 \pi} \int_{0}^{T} \frac{l(t)}{W(x(t), y(t))} d t .
\end{aligned}
$$

Recalling that $W(x(t), y(t)) \geq d-l_{1}$, we finally obtain that, for solutions $z(\cdot)$ with initial point $z_{0}=\left(x_{0}, y_{0}\right) \in \mathcal{C}_{1}^{s}$,

$$
\operatorname{rot}_{\psi_{2}}\left(T ; z_{0}\right)>\frac{M T}{2 \pi}-\frac{l_{1}}{2 \pi\left(d-l_{1}\right)} .
$$

Therefore, by the choice of $d$, we find that $\operatorname{rot}_{\psi_{2}}\left(T ; z_{0}\right)>j+\frac{1}{4}$, thus proving ii). $\square$

Proof of Lemma 12. Let $s$ be any sufficiently large number so that $\mathcal{C}_{1}^{s}$ is defined and let $c=g^{-1}(s)$ with $c$ in a left neighbourhood of $b$. After having fixed $s$, and hence $c$, for the rest of the proof, we consider the function $f:(-\infty, b[\rightarrow \mathbb{R}$ given by $f(x)=g(x)-s$. As usual, a solution of (14)-(17) will be a solution of $(\mathcal{E})_{s^{-}}(17)$. As $\gamma>0$ and $\lim _{x \rightarrow b^{-}} G(x)=+\infty$, the function $F(x)=\int_{c}^{x} f(\xi) d \xi$ satisfies (20) in both cases: $b=+\infty$ and $b<+\infty$. Moreover, by the first assumption in (28), we have $\lim \sup _{x \rightarrow-\infty} f(x)<+\infty$ and thus we can apply Corollary 5 and conclude that solutions of (14)-(17) are globally defined.

Choose $\alpha_{1}>\alpha \geq 0$ and $\beta^{*}>0$ such that $\beta^{*}>\beta$ if $\beta<+\infty$, while $\beta^{*}=+\infty$ if $\beta=+\infty$, satisfying

$$
\frac{f(x)}{x-c} \leq \alpha_{1}, \quad \forall x \leq c_{-} \quad \text { and } \quad \frac{f(x)}{x-c} \leq \beta^{*}, \forall x \geq c_{+}
$$

for some $c_{-}<c$ and $\left.c_{+} \in\right] c, b[$, and

$$
\left(\frac{2 \sqrt{\alpha_{1}} \sqrt{\beta^{*}}}{\sqrt{\alpha}_{1}+\sqrt{\beta}^{*}}\right)^{2}<\left(\frac{2 \pi k}{T}\right)^{2} .
$$

For $\beta=\beta^{*}=+\infty$, read the last inequality as $4 \alpha_{1}<\lambda_{k}$.

Let us define $\psi$ : $]-\infty,+\infty\left[\rightarrow \mathbb{R}\right.$ by $\psi(x)=\sqrt{\beta_{1}}(x-c)^{+}-\sqrt{\alpha_{1}}(x-c)^{-}$where $\beta_{1}=\beta^{*}$ if $\beta<+\infty$ and $\beta_{1}=1$ otherwise. The function $\psi$ is admissible with respect to $c$ and thus we can consider the corresponding system of modified polar coordinates. By Remark 1, in Section 2, we have $\delta_{\psi}=0$.

We also observe that there exist some positive constants $K$ and $L$ such that

$$
f(x) \psi(x) \leq \sqrt{\alpha_{1}} \psi(x)^{2}+K, \quad \forall x \leq c
$$

and if $\beta<+\infty$ and, necessarily, $b=+\infty$,

$$
f(x) \psi(x) \leq \sqrt{\beta_{1}} \psi(x)^{2}+L, \quad \forall x \geq c .
$$


Choose $R_{0}$ sufficiently large so that

$$
\frac{1}{\pi}\left(\frac{T \max \{K, L\}}{R_{0}^{2}}+\frac{l_{1}}{R_{0}}\right)<k-\frac{\sqrt{\alpha_{1}} \sqrt{\beta^{*}} T}{\pi\left(\sqrt{\alpha}_{1}+\sqrt{\beta}^{*}\right)} .
$$

We are in position now to construct the curve $\mathcal{C}_{2}^{s}$.

- If $\beta<+\infty$ we know that $b=+\infty$, that is, we can consider solutions of (14)-(17) with $t_{0}=0$ and $z_{0}=\left(x_{0}, y_{0}\right) \in \mathbb{R}^{2}$. Consider the compact set

$$
\mathcal{K}=\left\{(x, y) \in \mathbb{R}^{2}:\|(x-c, y)\| \leq \frac{R_{0}}{\min \left\{\sqrt{\alpha_{1}}, \sqrt{\beta_{1}}, 1\right\}}\right\} .
$$

By Lemma 10 we know that there exists a sufficiently large $R$ such that if $\|\left(x_{0}-\right.$ $\left.c, y_{0}\right) \| \geq R$ then $z(t)=(x(t), y(t)) \notin \mathcal{K}$ for every $t \in[0, T]$. We can also choose $R$ so large that $\max _{(x, y) \in \mathcal{C}_{1}^{s}}\|(x-c, y)\|<R$. Setting in this case

$$
\mathcal{C}_{2}^{s}=\{(x, y):\|(x-c, y)\|=R\},
$$

by the choice of $R$ we have $\mathcal{C}_{1}^{s} \subset \operatorname{int} \mathcal{C}_{2}^{s}$.

- Suppose now that $\gamma=+\infty$, so that $b<+\infty$ or $b=+\infty$ are both possible, and consider the compact set

$$
\mathcal{K}^{*}=\left\{(x, y) \in \mathbb{R}^{2}: x \leq c,\|(x-c, y)\| \leq \frac{R_{0}}{\min \left\{\sqrt{\alpha_{1}}, 1\right\}}\right\} .
$$

By Lemma 10 we know the existence of a compact $\mathcal{M} \subset]-\infty, b[\times \mathbb{R}$ such that if $z_{0}=\left(x_{0}, y_{0}\right) \notin \mathcal{M}$, then $z(t)=(x(t), y(t)) \notin \mathcal{K}^{*}$ for every $t \in[0, T]$. It suffices now to choose as $\mathcal{C}_{2}^{s}$ any simple closed curve $\left.\mathcal{C}_{2}^{s} \subset\right]-\infty, b[\times \mathbb{R}$ with

$$
\mathcal{M} \cup \mathcal{C}_{1}^{s} \subset \operatorname{int} \mathcal{C}_{2}^{s} \text {. }
$$

At this point, let us estimate the rotation number of the solutions starting from $\mathcal{C}_{2}^{s}$. The evaluation of the rotation will be performed by an implicit argument. More precisely, arguing by contradiction, we will show that the rotation cannot be larger than or equal to $k$.

Assume, by contradiction, that there exists a point $z_{0}=\left(x_{0}, y_{0}\right) \in \mathcal{C}_{2}^{s}$ such that $\operatorname{rot}_{\psi}\left(T ; z_{0}\right) \geq k$. Thus, for some $0<\tau \leq T$ we have $\operatorname{rot}_{\psi}\left(\tau ; z_{0}\right)=k$. Using $(7)$, the upper bound for $f \psi$ and recalling the definition of $\mathcal{K}$, (resp. $\left.\mathcal{K}^{*}\right)$, we obtain

$$
\begin{aligned}
k & =\frac{1}{\pi} \int_{\{t \in[0, \tau]: x(t)<c\}} \frac{\sqrt{\alpha_{1}} y(t)^{2}+f(x(t)) \psi(x(t))-w(t, x(t)) \psi(x(t))}{y(t)^{2}+\psi(x(t))^{2}} \\
& \leq \frac{\sqrt{\alpha_{1}}}{\pi} \operatorname{meas}\{t: x(t)<c\}+\frac{K T}{\pi R_{0}{ }^{2}}+\frac{l_{1}}{\pi R_{0}} .
\end{aligned}
$$

Similarly, if $\beta<+\infty$, we also get

$$
k \leq \frac{\sqrt{\beta_{1}}}{\pi} \text { meas }\{t: x(t)>c\}+\frac{T \max \{K, L\}}{\pi R_{0}^{2}}+\frac{l_{1}}{\pi R_{0}} .
$$

In the case $\beta=+\infty,(34)$ contradicts the choice of $R_{0}$ and the result follows. If $\beta<+\infty$, we can obtain, using (34) and (35),

$$
\left(\sqrt{\alpha_{1}}+\sqrt{\beta_{1}}\right) k \leq \frac{\sqrt{\alpha_{1}} \sqrt{\beta_{1}} T}{\pi}+\frac{T \max \{K, L\}}{\pi R_{0}^{2}}\left(\sqrt{\alpha_{1}}+\sqrt{\beta_{1}}\right)+\frac{l_{1}}{\pi R_{0}}\left(\sqrt{\alpha_{1}}+\sqrt{\beta_{1}}\right)
$$

and this once again contradicts the choice of $R_{0}$. So also in this case the result follows. 


\section{REMARKS AND RELATED RESUltS}

In this section we outline some possible variants of Theorem 7 and Theorem 8 for harmonic solutions which can be obtained with minor changes in the arguments of the preceding proofs.

First of all we describe what happens for the previous estimates when

$$
\gamma<\beta=+\infty
$$

In this situation, we have to follow the proof of Lemma 11 in the part we have already developed for the case $\gamma=+\infty$, where we proved that $\operatorname{rot}_{\psi_{2}}\left(T ; z_{0}\right)>j+\frac{1}{4}$ if $g^{\prime}(x)>M^{2}$, for $x \geq a_{1}^{j}$, with $M>\frac{2 \pi}{T}\left(j+\frac{1}{4}\right)$ and $z_{0}$ is an arbitrary point belonging to $\mathcal{C}_{1}^{s}$, a strictly star-shaped curve around $\left(g^{-1}(s), 0\right)$. Now, using Lemma 12 which remains unchanged, we can repeat verbatim the proofs of the main results (Theorem 7 and Theorem 8) if

$$
4 \alpha<(2 \pi k / T)^{2}<(\pi(4 j+1) / 2 T)^{2}<\gamma
$$

holds, with the caution of taking $\alpha=0$ for the case when (30) is satisfied.

Then the following theorem can be stated in which we assume a condition on the eigenvalues which guarantees the validity of (37).

Theorem 9. Assume $a=-\infty,(28)$, (29) and (36). Suppose that $\lim _{x \rightarrow b^{-}} G(x)=$ $+\infty$, where $G$ is a primitive of $g$ and let $k, j \in \mathbb{Z}_{0}^{+}$be such that

$$
4 \alpha<\lambda_{k}<\lambda_{j+1}<\gamma
$$

Then for each integer $r$, with $k \leq r \leq j$, equation $(E)_{s}$ has at least two T-periodic solutions $x_{i}(\cdot), i=1,2$, such that $x_{i}(\cdot)-g^{-1}(s)$ has exactly $2 r$ zeros for each $s$ sufficiently large. The same conclusion holds, taking $\alpha=0$ and $k=1$, if we assume $a \geq-\infty$ and (30) instead of (28).

At this point we discuss separately the case in which $g$ is defined on the whole real line and the singularity cases.

6.1. The real line as a domain. Suppose that $g: \mathbb{R} \rightarrow \mathbb{R}$ is a continuously differentiable function such that

$$
\limsup _{x \rightarrow-\infty} g^{\prime}(x):=\alpha_{0}, \quad \gamma_{0}:=\liminf _{x \rightarrow+\infty} g^{\prime}(x) \leq \limsup _{x \rightarrow+\infty} g^{\prime}(x):=\beta_{0},
$$

with

$$
\max \left\{\alpha_{0}, 0\right\}<\gamma_{0} \leq \beta_{0} \leq+\infty
$$

Let $\lambda_{n}$ be the $n$-th positive eigenvalue for the differential operator $-u^{\prime \prime}$ with the $T$-periodic boundary conditions (recall that $\lambda_{n}=(2 \pi / T)^{2} n^{2}$ with $n \in \mathbb{Z}^{+}$). If $\gamma_{0}=\beta_{0}=+\infty$, then we can apply Theorem 7 with $\alpha=\max \left\{\alpha_{0}, 0\right\}, \gamma=\gamma_{0}$ and $\beta=\beta_{0}$ in the case that $\lim \sup _{x \rightarrow-\infty} g(x)<+\infty$, or we can apply Theorem 8 if $\lim \sup _{x \rightarrow-\infty} g(x)=+\infty$. Then anyway we can obtain: 
Corollary 6. Suppose that $\lim \sup _{x \rightarrow-\infty} g^{\prime}(x)<\lim _{x \rightarrow+\infty} g^{\prime}(x)=+\infty$. Then, for each $n \in \mathbb{Z}_{0}^{+}$with $\lambda_{n}>4 \max \left\{\alpha_{0}, 0\right\}$, there is $s_{n}$ such that for each $s>s_{n},(E)_{s}$ has at least two harmonic solutions crossing $2 n$ times the value $g^{-1}(s)$ in the interval $[0, T[$.

Corollary 6 corresponds to an improved version of [43, Theorem 1] for the periodic case. Indeed, in [43, Theorem 1] the existence of a limit for $g^{\prime}(x)$ at $-\infty$ was required.

If $\gamma_{0} \leq \beta_{0}<+\infty$, then we can apply Theorem 7 with $\alpha=\max \left\{\alpha_{0}, 0\right\}, \gamma=\gamma_{0}$ and $\beta=\beta_{0}$, or Theorem 8 as above, in order to have:

Corollary 7. Suppose that

$$
\limsup _{x \rightarrow-\infty} g^{\prime}(x)=\alpha_{0}, \quad 0<\gamma_{0}=\liminf _{x \rightarrow+\infty} g^{\prime}(x) \leq \limsup _{x \rightarrow+\infty} g^{\prime}(x)=\beta_{0}
$$

and let $l$ be the integer part of $(T / \pi)\left(\sqrt{\alpha \beta_{0}} /\left(\sqrt{\alpha}+\sqrt{\beta_{0}}\right)\right)$, with $\alpha=\max \left\{\alpha_{0}, 0\right\}$. If there exists an integer $n>l$, such that $\lambda_{n}<\gamma$ then equation $(E)_{s}$ has at least $2(n-l)$ harmonic solutions for each sufficiently large positive $s$.

Corollary 7 improves the conditions on the limits for $g^{\prime}(x)$ as $x \rightarrow \pm \infty$ considered in $[8$, Theorem $1.2(\mathrm{a})]$. Indeed, in [8] the existence of $2(n-l)+1$ harmonic solutions for large positive $s$ is proved under the more restrictive condition that limits for $g^{\prime}(x)$ do exist with $\alpha_{0}>\lambda_{k-1}$ for some $k \leq n$. Moreover, in [8] it is required also that $\lambda_{n}<\gamma_{0}=\beta_{0}<\lambda_{n+1}$. It is not difficult to see that if we also require the "nonresonance" condition $\left[\gamma_{0}, \beta_{0}\right] \cap\left\{\lambda_{j}: j \in \mathbb{Z}_{0}^{+}\right\}=\emptyset$, then we can prove the existence of a further $T$-periodic solution for $s>0$ and large and therefore we can obtain $2(n-l)+1$ harmonic solutions too.

If $g$ is unbounded from above on $(-\infty, 0]$, then according to Theorem 8 , the previous results hold without any condition on $\alpha_{0}, \gamma_{0}$ and $\beta_{0}$, except $\gamma_{0}>0$. In this case, however, more precise information about the solutions can be further derived. Indeed, in [7, Corollary II.1.9] it is proved that if $g(x) \rightarrow+\infty$ as $x \rightarrow-\infty$, then equation $(E)_{s}$ has at least one (negative) $T$-periodic solution for $s$ positive and large. Such a solution is found between a constant lower solution $\underline{x}$ and a constant upper solution $\bar{x}$ with $\underline{x}<\bar{x}<<0$. On the other hand, the solutions $x(t)$ we find via the Poincaré - Birkhoff fixed point theorem cross $g^{-1}(s)>0$, for large $s>0$ and therefore we can conclude that our solutions are not the same found in [7, Corollary II.1.9]. Hence, if $g(-\infty)=+\infty$, we have another solution to add to our collection. Thus we can state the following corollaries:

Corollary 8. Suppose that

$$
\lim _{x \rightarrow-\infty} g(x)=\lim _{x \rightarrow+\infty} g^{\prime}(x)=+\infty
$$

Then, for each $n \in \mathbb{Z}_{0}^{+}$, there is $s_{n}$ such that $(E)_{s}$ has at least $2 n+1$ harmonic solutions for each $s>s_{n}$. Among these solutions one is negative and, for each integer $j$ with $1 \leq j \leq n$, there are two solutions with $x(\cdot)-c$ having exactly $2 j$ zeros in the interval $[0, T[$ where $g(c)=s$ for $c>0$. 
Corollary 9. Suppose that $\lim _{x \rightarrow-\infty} g(x)=+\infty$ and let $n \in \mathbb{Z}_{0}^{+}$be such that

$$
0<\lambda_{n}<\gamma_{0}=\liminf _{x \rightarrow+\infty} g^{\prime}(x) \leq \limsup _{x \rightarrow+\infty} g^{\prime}(x)=\beta_{0}<+\infty .
$$

Then there is $s_{n}$ such that $(E)_{s}$ has at least $2 n+1$ harmonic solutions for each $s>$ $s_{n}$. Among these solutions one is negative and, for each integer $j$ with $1 \leq j \leq n$, there are two solutions with $x(\cdot)-c$ having exactly $2 j$ zeros in the interval $[0, T[$ where $g(c)=s$ for $c>0$.

The proof of Corollaries 8 and 9 follows immediately from Theorem 8 and the previous remark about the result in [7]. Notice that, if in Corollary $9,\left[\gamma_{0}, \beta_{0}\right]$ does not contain eigenvalues, then a further $T$-periodic solution appears and thus we can conclude with the existence of $2 n+2$ harmonic solutions like in [8, Theorem 1.1]. It is possible to check that Corollaries 8 and 9 hold true if we replace the condition for $g(x)$ at $-\infty$, with $\lim \sup _{x \rightarrow-\infty} g(x)=+\infty$.

6.2. The singularity cases. Suppose that $g:] a, b[\rightarrow \mathbb{R}$, with $] a, b[\neq \mathbb{R}$ is a continuously differentiable function. Let $G$ denote a primitive of $g$ on $] a, b[$.

We concentrate our discussion on the case $-\infty<a$ since the situation when $a=-\infty$ is clear from the previous theorems. Recall that for $a \in \mathbb{R}$, we have to assume

$$
\limsup _{x \rightarrow a^{+}} g(x)=+\infty
$$

for the validity of the truncation argument. On the other hand, arguing directly on the original equation with the method of lower and upper solutions, it is possible to find another $T$-periodic solution in a right neighbourhood of $a$. Hence we can restate Corollary 9 for a function $g:] a,+\infty) \rightarrow \mathbb{R}$, as follows:

Corollary 10. Suppose that $\lim \sup _{x \rightarrow a^{+}} g(x)=+\infty$ and let $n \in \mathbb{Z}_{0}^{+}$be such that

$$
0<\lambda_{n}<\gamma_{0}=\liminf _{x \rightarrow+\infty} g^{\prime}(x) \leq \limsup _{x \rightarrow+\infty} g^{\prime}(x)=\beta_{0}<+\infty .
$$

Then there is $s_{n}$ such that $(E)_{s}$ has at least $2 n+1$ harmonic solutions for each $s>s_{n}$. Among these solutions one lies on a right neighbourhood of a and, for each integer $j$ with $1 \leq j \leq n$, there are two solutions with $x(\cdot)-c$ having exactly $2 j$ zeros in the interval $[0, T[$ where $g(c)=s$ for $c>0$.

Also in this case, we can find at least $2 n+2$ harmonic solutions if there are no eigenvalues between $\gamma_{0}$ and $\beta_{0}$.

For a function $g:] a, b[\rightarrow \mathbb{R}$, with $b \leq+\infty$, we have the following variant of Corollaries 6 and 8 .

Corollary 11. Suppose that $\lim \sup _{x \rightarrow a^{+}} g(x)=+\infty$ and

$$
\lim _{x \rightarrow b^{-}} g^{\prime}(x)=\lim _{x \rightarrow b^{-}} G(x)=+\infty .
$$

Then, for each $n \in \mathbb{Z}_{0}^{+}$, there is $s_{n}$ such that $(E)_{s}$ has at least $2 n+1$ harmonic solutions for each $s>s_{n}$. Among these solutions one lies on a right neighbourhood of $a$ and, for each integer $j$ with $1 \leq j \leq n$, there are two solutions with $x(\cdot)-c$ having 
exactly $2 j$ zeros in the interval $[0, T[$ where $g(c)=s$ for $c$ in a left neighbourhood of $b$.

Corollary 10 extends [8, Theorem 1.1] to the case of a singularity of "attractive type" in $a$, while Corollary 11 may be seen as an improved version of Zinner's theorem to a periodic problem with also the possibility to treat a double singularity when $b<+\infty$.

We stop for the moment with the list of other possible consequences of our main result, passing now to the discussion of the problem concerning subharmonic solutions.

\section{ExistenCE OF SUbHARMONIC SOlUtions}

In this section we are interested in the existence of other kinds of periodic solutions for equation $(E)_{s}$ under conditions (15), (28) or (30), and (29). First of all we observe that, according to [38], we cannot prevent the possibility that $(E)_{s}$ may possess periodic solutions whose period is uncommensurable with $T$. However, we note that such a situation will never occur when $w$ takes the form of $w=w(t)$ with $T>0$ as minimal period. In this case, it is also clear that all the possible periodic solutions of $(E)_{s}$ will have periods multiple of $T$ (subharmonics). Thus, with the aim of obtaining a result which is applicable to the simpler case of a forcing term $w(t)$ independent on the $x$-variable, we prefer to restrict ourselves to the consideration of subharmonic solutions. Following [38], we call subharmonic solution of order $m \geq 2$, a periodic solution whose minimal period in the set $\left\{j T: j \in \mathbb{Z}_{0}^{+}\right\}$ is $m T$. As remarked above, this does not mean that $m T$ is effectively the minimal period. The minimality of the period can be guaranteed by additional assumptions like $\left(W^{*}\right)$ considered in the introduction.

We also recall that if $x(\cdot)$ is a subharmonic solution of order $m$, then the $m-1$ functions $x_{i}(\cdot)=x(\cdot+i T), i=1, \ldots, m-1$, are also subharmonics of order $m$, pairwise distinct and distinct from $x(\cdot)$. We consider these translates in time as equivalent to $x(\cdot)$ (they belong to the same periodicity class).

We have the following theorem that we state in the case of a function $g:] a, b[\rightarrow \mathbb{R}$ which is of class $\mathcal{C}^{1}$. Note that we take $a=-\infty$ in the case when (28) is considered.

Theorem 10. Assume conditions (15), (28) or (30) and (29) and suppose that $\lim _{x \rightarrow b^{-}} G(x)=+\infty$ where $G$ is a primitive of $g$. Then we have:

- If $\gamma<+\infty$ and

$$
\frac{2 \sqrt{\alpha} \sqrt{\beta}}{\sqrt{\alpha}+\sqrt{\beta}}<\sqrt{\gamma}
$$

then for each $k \in \mathbb{Z}_{0}^{+}$there exists $m_{k}$ such that for every $m \geq m_{k},(E)_{s}$ has at least $2 k$ subharmonic solutions of order $m$ which do not belong to the same periodicity class, for each sufficiently large positive $s$, say $s>s\left(l_{1}, k, m\right)>0$ (Read $\alpha=0$ in case we substitute (28) with (30).)

- If $\gamma=+\infty$, for each $m>1$, there exists an arbitrarily large number of $m$-th order subharmonic solutions of equation $(E)_{s}$, for sufficiently large s. More precisely, for each $m>1$, there exists $n_{m}$ such that for each $n \geq n_{m}$ prime with $m$, there is a $s_{n}=s\left(l_{1}, m, n\right)>0$ which satisfies: for every $s>s_{n}$ there exist at least two subharmonic solutions of order $m$ to $(E)_{s}$ which do 
not belong to the same periodicity class. Moreover, denoting by $x_{i}(\cdot), i=1,2$, these solutions, $x_{i}(\cdot)-g^{-1}(s)$ has exactly $2 n$ zeros on the interval $[0, m T[$.

Proof. The proof follows a similar argument as in Theorems 7 and 8, working in the interval $[0, m T]$, instead of the interval $[0, T]$. We consider at first the case which is analogous to Theorem 7 and therefore we assume (28).

Suppose at first that $\gamma<+\infty$. Let us fix two positive constants $A$ and $B$ such that

$$
\frac{T \sqrt{\alpha} \sqrt{\beta}}{\pi(\sqrt{\alpha}+\sqrt{\beta})}<A<B<\frac{T \sqrt{\gamma}}{2 \pi}
$$

and take a positive integer $m^{*}$ such that

$$
\left(m^{*}\right)^{-1}<\frac{T \sqrt{\gamma}}{2 \pi}-B
$$

Fix $k \in \mathbb{Z}_{0}^{+}$and consider the $2 k$ disjoint intervals $] A+\ell \frac{B-A}{2 k}, A+(\ell+1) \frac{B-A}{2 k}[$, with $\ell=0, \ldots, 2 k-1$.

Claim: There exists an $m_{k}^{*} \in \mathbb{Z}_{0}^{+}$with $m_{k}^{*} \geq m^{*}$ such that for every $m \geq m_{k}^{*}$ and $\ell \in\{0, \ldots, 2 k-1\}$ even, there exists at least an integer $n^{\ell}$, which is prime with $m$ and such that

$$
\left.\frac{n^{\ell}}{m} \in\right] A+\ell \frac{B-A}{2 k}, A+(\ell+2) \frac{B-A}{2 k}[.
$$

Indeed, choose an arbitrary $\ell$ as above and observe that $A+\ell \frac{B-A}{2 k}<A+$ $(\ell+2) \frac{B-A}{2 k}$. Adapting to the present setting the argument used in $[13$, proof of Th.2.3], we can prove by the "Prime Number Theorem" [24] that there exists a constant $m_{\ell} \in \mathbb{Z}_{0}^{+}$with $m_{\ell}>m^{*}$ such that for every integer $m>m_{\ell}$ there exist two prime integers $n_{1}^{\ell}(m)$ and $n_{2}^{\ell}(m)$ such that

$$
\left.n_{1}^{\ell}(m) \in\right]\left(A+\ell \frac{B-A}{2 k}\right) m,\left(A+(\ell+1) \frac{B-A}{2 k}\right) m[
$$

and

$$
\left.n_{2}^{\ell}(m) \in\right]\left(A+(\ell+1) \frac{B-A}{2 k}\right) m,\left(A+(\ell+2) \frac{B-A}{2 k}\right) m[.
$$

We prove that $n_{1}^{\ell}(m)$ is prime with $m$ or $n_{2}^{\ell}(m)$ is prime with $m$ for $m>m_{\ell}$. In fact, if this is not the case, $n_{1}^{\ell}(m) n_{2}^{\ell}(m)$ divides $m$, so that $n_{1}^{\ell}(m) n_{2}^{\ell}(m) \leq m$. Moreover, we know that $n_{1}^{\ell}(m) n_{2}^{\ell}(m)>(A m)^{2}$ and so we obtain a contradiction for $m$ sufficiently large $\left(m>A^{-2}\right)$. Take now $m_{k}^{*}>\max \left\{m_{\ell}: \ell=0, \ldots, 2 k-1\right\}$ with also $m_{k}^{*}>A^{-2}$ and, every time when $m \geq m_{k}^{*}$, choose as $n^{\ell}$ one of the two numbers $n_{1}^{\ell}(m)$ and $n_{2}^{\ell}(m)$ which is prime with $m$. Hence the claim follows.

Now, let $m \geq m_{k}^{*} \geq m^{*}$ and consider the $n^{\ell}$ given by the claim. By the original choice of $A, B$ and $m^{*}$, we have that

$$
\frac{2 \sqrt{\alpha} \sqrt{\beta}}{\sqrt{\alpha}+\sqrt{\beta}}<\frac{2 \pi n^{\ell}}{m T}<\frac{2 \pi\left(n^{\ell}+1\right)}{m T}<\sqrt{\gamma} .
$$

Since $w(\cdot, x)$ which is $T$-periodic is also $m T$-periodic, then we can apply Theorem 7 (if $\beta<+\infty$ ) or Theorem 9 (if $\beta=+\infty$ ), for the search of $m T$-periodic solutions. Note that the positive eigenvalues for the $m T$-periodic problem take the 
form $(2 \pi i / m T)^{2}$, for $i \in \mathbb{Z}_{0}^{+}$and replace the constant $l_{1}$ with $l_{m}=m l_{1}$ in all the corresponding estimates which appear in the proofs of the auxiliary lemmas. Therefore we can conclude that for each sufficiently large $s$, (say $s>s\left(l_{1}, k, m\right)$ ) and for each $\ell$ even in $\{0, \ldots, 2 k-1\}$, there exist two $m T$-periodic solutions $x_{i, \ell}(\cdot)$, $i=1,2$, of equation $(E)_{s}$ such that $x_{i, \ell}(\cdot)-g^{-1}(s)$ has exactly $2 n^{\ell}$ zeros in the interval $[0, m T[$.

Let us check now that $m T$ is the minimal period of each of these functions on the set $\left\{j T: j \in \mathbb{Z}_{0}^{+}\right\}$. Indeed, suppose by contradiction that $x_{i, \ell}(\cdot)$ is $j T$-periodic with $1 \leq j<m$. The number of turns (rotation number) of $z_{i, \ell}(\cdot)=\left(x_{i, \ell}(\cdot), x_{i, \ell}^{\prime}(\cdot)\right.$ ) around the point $\left(g^{-1}(s), 0\right)$ on the interval [0,mT is $n^{\ell}$. Let $r^{\ell}$ be the number of turns on the interval $\left[0, j T\left[\right.\right.$. Clearly, the number of turns of $z_{i, \ell}(\cdot)$ on the interval $\left[0, m j T\right.$ [ will be $n^{\ell} j=r^{\ell} m$. This last identity is a contradiction with the fact that $n^{\ell}$ is prime with $m$. Hence we have proved the existence of two subharmonics of order $m$ for each $\ell$. By Theorem 5 we know that $x_{1, \ell}(\cdot)$ and $x_{2, \ell}(\cdot)$ do not belong to the same periodicity class for each fixed $\ell$. On the other hand, solutions with different number of zeros cannot belong to the same periodicity class. Therefore the $2 k$ subharmonic solutions of order $m, x_{i, \ell}(\cdot)$, are mutually non-equivalent.

At last, suppose that $\gamma=+\infty$. Let $m \in \mathbb{Z}_{0}^{+}$be chosen arbitrarily. Take an integer $n_{m}$ such that $4 \alpha<\left(2 \pi n_{m} / m T\right)^{2}$. Let $n \geq n_{m}$ be an integer prime with $m$. We can apply Theorem 7 for the search of $m T$-periodic solutions and conclude that for each sufficiently large $s$ (say $s>s\left(l_{1}, m, n\right)$ ), there exist two $m T$-periodic solutions $x_{i}(\cdot), i=1,2$, of equation $(E)_{s}$, which do not belong to the same periodicity class, such that $x_{i}(\cdot)-g^{-1}(s)$ has exactly $2 n$ zeros on the interval [0,mT[. As above, we conclude that these solutions are subharmonics of order $m$.

It remains to consider the case in which we assume (30) instead of (28), allowing both the possibilities: $a=-\infty$ or $a>-\infty$. The proof follows precisely the same lines as above, with the only difference that now we have to invoke Theorem 8 or Theorem 9 for the existence of $m T$-periodic solutions.

Note that Theorem 10 covers Theorem 3 in the Introduction. Moreover, combining Theorem 7 with Theorem 10, we can also obtain Theorem 1 in the Introduction.

Remark 4. It seems worth noticing the following points:

- Theorem 1 and Theorem 2 in the Introduction follow immediately from Theorem 7 and Theorem 8, respectively, arguing as in the last part of the proof of Theorem 10. Indeed, thinking at the map $w(\cdot, x)$ as an $m T$-periodic function, for some $m>1$, we can obtain the existence of $m T$-periodic solutions $x(\cdot)$ to $(E)_{s}$ provided that the hypotheses of Theorem 7 or Theorem 8 are satisfied with respect to the new period $m T$. In this case, the only problem we have to discuss is about the minimality of the period for $x(\cdot)$ in the class $\{j T: j=1, \ldots, m\}$. From Theorem 7 and Theorem 8 we also know that the solutions we find have a precise number of rotation, or, equivalently, $x(\cdot)-g^{-1}(s)$ has a number of zeros which can be determined exactly. Indeed, from the above recalled theorems and under the assumptions on the coefficients $\alpha, \beta, \gamma$ and $k$, we find two solutions $x_{i}(\cdot)$ (for $i=1,2)$, of period $m T$ and such that $x_{i}(\cdot)-g^{-1}(s)$ has precisely $2 k$ zeros in the interval $[0, m T$. Now, arguing as in the proof of Theorem 10, we can conclude that the minimal period of $x_{i}(\cdot)$ is precisely $m T$, if $k$ and $m$ are co-prime integers.

- All the remarks in Section 6, which are pertinent to the present setting for the search of subharmonics, can be applied as well. The only point in which we 
cannot guarantee the minimality of the period is for the supplementary solutions which are found without the use of the Poincaré - Birkhoff theorem (like, e.g. those we obtain using the method of lower and upper solutions).

\section{FinAL REMARKS}

A last point which has been left out until now is to see what happens if we interchange the behaviour of the function $g$ at the extreme points of its domain. More precisely, assume that $g:] a, b\left[\rightarrow \mathbb{R}\right.$ is of class $\mathcal{C}^{1}$ and let $G$ be a primitive of $g$. Then we have the next results which are, in some sense, dual with respect to Theorem 7, Theorem 8 and Theorem 10.

Assume that $w$ satisfies condition (15) and suppose that one of the following two assumptions hold:

$$
b \leq+\infty \quad \text { and } \liminf _{x \rightarrow b^{-}} g(x)=-\infty,
$$

or

$$
b=+\infty, \quad \liminf _{x \rightarrow+\infty} g(x)>-\infty \text { and } \limsup _{x \rightarrow+\infty} g^{\prime}(x) \leq \alpha<\infty,
$$

with $\alpha \geq 0$. Assume also that

$$
0<\gamma \leq \liminf _{x \rightarrow a^{+}} g^{\prime}(x) \leq \limsup _{x \rightarrow a^{+}} g^{\prime}(x) \leq \beta \leq+\infty .
$$

In comparison with Theorem 7 and Theorem 8, we can state:

Theorem 11. Suppose that (42) and (40) or (41) hold. Assume $\lim G(x)=$ $+\infty$, where $G$ is a primitive of $g$ and let $k \in \mathbb{Z}_{0}^{+}$be such that $\left(\frac{2 \sqrt{\alpha} \sqrt{\beta}}{\sqrt{\alpha}+\sqrt{\beta}}\right)^{2}<a^{+}$ the convention that $4 \alpha<\lambda_{k}$ when $\beta=+\infty$ and $\alpha=0$ in case that (40) is assumed. Then we have:

- If $\beta<+\infty$ and $\lambda_{j}<\gamma$, for some integer $j \geq k$, then equation $(E)_{s}$ has at least $2(j-k+1)$ harmonic solutions for each sufficiently large $|s|$ with $s<0$. More precisely, for each $s$ negative with $|s|$ large and for $k \leq r \leq j$ there exist two T-periodic solutions $x_{i}(\cdot), i=1,2$, of $(E)_{s}$, such that for each $i$, $x_{i}(\cdot)-g^{-1}(s)$ has exactly $2 r$ zeros in the interval $[0, T[$.

- If $\gamma=+\infty$, for each integer $j \geq k$ equation $(E)_{s}$ has at least $2(j-k+1)$ harmonic solutions for each sufficiently large $|s|$ with $s<0$. More precisely, for each $s$ negative with $|s|$ large and each $k \leq r \leq j$, there exist two $T$ periodic solutions $x_{i}(\cdot), i=1,2$, of $(E)_{s}$, such that for each $i, x_{i}(\cdot)-g^{-1}(s)$ vanishes exactly $2 r$ times in the interval $[0, T[$.

Similarly to Theorem 10, we have:

Theorem 12. Suppose that (42) and (40) or (41) hold. Assume that $\lim _{x \rightarrow a^{+}} G(x)=$ $+\infty$, where $G$ is a primitive of $g$. Then we have:

- If $\gamma<+\infty$ and

$$
\frac{2 \sqrt{\alpha} \sqrt{\beta}}{\sqrt{\alpha}+\sqrt{\beta}}<\sqrt{\gamma}
$$


then for each $k \in \mathbb{Z}_{0}^{+}$there exists $m_{k}$ such that for every $m \geq m_{k},(E)_{s}$ has at least $2 k$ subharmonic solutions of order $m$ that do not belong to the same periodicity class, for each $s$ negative with $|s|$ sufficiently large. (Read $\alpha=0$ in (43) in case that (40) is assumed.)

- If $\gamma=+\infty$, for each $m>1$, there exists an arbitrarily large number of $m$-th order subharmonic solutions of equation $(E)_{s}$, for each $s$ negative with $|s|$ sufficiently large. More precisely, for each $m>1$, there exists $n_{m}$ such that for each $n \geq n_{m}$ prime with $m$, there are at least two subharmonic solutions of order $m$ to $(E)_{s}$ that do not belong to the same periodicity class, for $s$ negative with $|s|$ large. Moreover, denoting by $x_{i}(\cdot), i=1,2$, these solutions, $x_{i}(\cdot)-g^{-1}(s)$ has exactly $2 n$ zeros in the interval $[0, m T[$.

Theorems 11 and 12 follow from the corresponding Theorems 7 and 10 and Theorem 8 applied to the differential equation

$$
u^{\prime \prime}+\tilde{g}(u)=(-s)+\tilde{w}(t, u)
$$

where $\tilde{g}(x)=-g(-x)$ and $\tilde{w}(t, x)=-w(t,-x)$. Observe that $\tilde{g}(x)$ and $\tilde{w}(t, x)$ are defined in $]-b,-a[$. A straightforward computation shows that $x(\cdot)$ is a solution of $(E)_{s}$ if and only if $u(\cdot)=-x(\cdot)$ is a solution of $(\tilde{E})_{s}$. The same trick can be used to have a dual version of Theorem 9 .

All the results in Section 6 which were derived from Theorem 7 and Theorem 8 can be stated in the corresponding dual version. In this manner, also [8, Theorem $1.2(\mathrm{~b})]$ can be derived.

Now, putting together all the results given in Sections 4, 6, 7 and 8 with the above ones, it is possible to derive some conclusions in which we can consider a rather exhaustive set of hypotheses.

Corollary 12. Let $G:] a, b\left[\rightarrow \mathbb{R}\right.$ be a function of class $\mathcal{C}^{2}$ with $G^{\prime}=g$, such that

$$
\left|G\left(a^{+}\right)\right|=\left|G\left(b^{-}\right)\right|=\left|g^{\prime}\left(a^{+}\right)\right|=\left|g^{\prime}\left(b^{-}\right)\right|=+\infty \quad \text { and } \quad g^{\prime}\left(a^{+}\right) g^{\prime}\left(b^{-}\right)<0 \text {. }
$$

Then, for each $m \in \mathbb{Z}_{0}^{+}$, there exists an arbitrarily large number of $m$-th order subharmonic solutions of equation $(E)_{s}$, for an unbounded interval of $s$. More precisely, for each $m \geq 1$, there exists a $n_{m}$ such that for each $n \geq n_{m}$ prime with $m$, there is a $s_{n}>0$ which verifies that either for $s>s_{n}$ or for $s<-s_{n}$, there exist at least two subharmonic solutions of order $m$ to $(E)_{s}$ which do not belong to the same periodicity class and oscillate around $g^{-1}(s)$, having precisely $2 n$ crossings with $g^{-1}(s)$ in the interval $\left[0, m T\left[\right.\right.$. We can discriminate whether $s>s_{n}$ or $s<-s_{n}$, according to the fact that $g^{\prime}\left(b^{+}\right)=+\infty$ or $g^{\prime}\left(b^{+}\right)=-\infty$.

Corollary 13. Let $g: \mathbb{R} \rightarrow \mathbb{R}$ be a function of class $\mathcal{C}^{1}$ such that the limits $g^{\prime}(-\infty)$ and $g^{\prime}(+\infty)$ exist and are finite. Assume that $g^{\prime}(-\infty) \neq g^{\prime}(+\infty)$ and suppose that $\max \left\{g^{\prime}(-\infty), g^{\prime}(+\infty)\right\}:=g^{*}>0$. Then, for each $k \in \mathbb{Z}_{0}^{+}$, there exists $m_{k}$ such that for every $m \geq m_{k},(E)_{s}$ has at least $2 k$ subharmonic solutions of order $m$ which are not in the same periodicity class, for each s belonging to an unbounded interval. We can discriminate whether the parameter $s$ has to be chosen in a neighborhood of $+\infty$ or in a neighborhood of $-\infty$, according to the fact that $g^{\prime}(+\infty)=g^{*}$ or $g^{\prime}(-\infty)=g^{*}$.

Remark 5. We describe the conclusions which can be drawn for the case in which the uniqueness of the solutions for the Cauchy problems is not guaranteed. Precisely, 
suppose that $w(t, x)$ is continuous and bounded on $\mathbb{R} \times] a, b[$ (not necessarily locally lipschitzian) and $g:] a, b[\rightarrow \mathbb{R}$ is continuous. In this case the results concerning the global existence of the solutions are still valid without any change. At this point, one could use the smoothing and approximation argument described for the same context in [11] and apply each time the Poincaré - Birkhoff theorem to the approximating planar systems depending on a index $n$. Hence it is possible to prove that each time there are two fixed points, say $z_{n}^{1}$ and $z_{n}^{2}$ for the Poincaré map (or for its iterates in case of the search of subharmonics) and all these fixed points belong to the same annulus, thanks to some uniformity in the estimates. Then, passing to the limit on two subsequences of fixed points taken from $\left(z_{n}^{1}\right)_{n}$ and $\left(z_{n}^{2}\right)_{n}$, converging respectively to $z_{1}^{*}$ and $z_{2}^{*}$, we can see that periodic solutions exist also for the original equation. These periodic solutions will satisfy $\left(x(0), x^{\prime}(0)\right)=z_{i}^{*}$ $(i=1,2)$. However, we have no way to guarantee that $z_{1}^{*} \neq z_{2}^{*}$ and therefore, we can conclude just finding at least one periodic solution with initial point in the annulus, for the given equation, whenever at least two periodic solutions previously occurred in the smooth case. All this expensive procedure makes sense only with respect to the benefit of relaxing some restrictions on $g$ and $w$ we had to consider before. In particular, one can see that in the results of Sections 4-7 one could assume $g$ of class $\mathcal{C}^{1}$ only on a left neighbourhood of $b$ and relax the assumptions on $\limsup g^{\prime}(x)$ in (28) to $\lim \sup _{x \rightarrow-\infty} \frac{g(x)}{x} \leq \alpha$. Also in (29) for the subcase $b=+\infty$ one can take $\lim \sup _{x \rightarrow+\infty} \frac{g(x)}{x} \leq \beta$. Similar changes can be done to improve (41) and (42), assuming $g$ of class $\mathcal{C}^{1}$ only in a right neighbourhood of $a$. We leave to the interested reader the care of restating the corresponding corollaries under these slightly more general assumptions taking into account the "loss" in the resulting number of periodic solutions.

We conclude this section and the paper with a choice of examples showing the range of applicability of our results with respect to some previous ones.

Example 11. As a starting point, we consider the equation

$$
x^{\prime \prime}+c(x) x^{\prime}+x^{2}=s+e(t),
$$

examined by Fabry, Mawhin and Nkashama in [15]. In [15] it is proved that, for any continuous function $c(x),(44)$ has at least two periodic solutions having the same period of $e(t)$, for $s>0$ and sufficiently large. We consider now the case $c(x) \equiv 0$, so that (44) takes the form

$$
x^{\prime \prime}+x^{2}=s+e(t) .
$$

With respect to (45) we recall also a result of Lupo, Solimini and Srikanth in [29] where, for the corresponding two-point BVP (and the slightly modified forcing term), the number of the solutions increases as $s \rightarrow+\infty$ (see also [43] for a recent contribution in this direction). In this case, assuming $e(\cdot)$ continuous and periodic with minimal period $T>0$, we can apply Corollary 8 and conclude that for any positive integer $n$, there is a constant $s_{n}$ such that equation (45) has at least $2 n+$ 1 harmonic solutions for all $s>s_{n}$. One of these $T$-periodic solutions, like in [15] and [7], lies between a constant lower solution and a constant upper solution which are both negative, while the remaining $2 n$ solutions oscillate around $\sqrt{s}$ with 
respectively $2,4, \ldots, 2 n$ crossings of $\sqrt{s}$ in the interval [0,T[. Moreover, according to Theorem 10, we know that for each pair of positive integers, $m$ and $n$, we can find a constant $s_{m, n}>0$ such that equation (45) has at least $2 n$ periodic solutions of minimal period $m T$ for all $s>s_{m, n}$. These solutions oscillate around $\sqrt{s}$ respectively $2 j_{1}, 2 j_{2}, \ldots, 2 j_{n}$ times in the interval [0, $m T$ [, where $j_{1}<j_{2}<\ldots<j_{n}$ is a finite sequence of integers which are prime with $m$.

Example 2. As a second example, we consider a case of jumping nonlinearities for the periodically perturbed equation

$$
x^{\prime \prime}+g(x)=s+e(t)
$$

with $e(\cdot)$ satisfying the same conditions as above. We also assume for $a \geq-\infty$, that $g:] a,+\infty) \rightarrow \mathbb{R}$ is a continuously differentiable function such that

$$
g^{\prime}\left(a^{+}\right)=G\left(a^{+}\right)=+\infty
$$

where $G$ is a primitive of $g$ in the interval $] a,+\infty)$. Suppose now that

$$
\lim _{x \rightarrow+\infty} g^{\prime}(x)=\lambda<+\infty
$$

If $\lambda>0$, the above conditions permit to enter in a setting already discussed by Figueiredo and Ruf in [16], Fabry and Habets in [14] for the case $a=-\infty$ and by del Pino, Manásevich and Montero in [9], for the case $a=0$. As a consequence of these quoted results, equation (46) has at least one $T$-periodic solution for each $s$, provided that $\lambda \neq(\pi k / T)^{2}$ for all positive integers $k$. Here we can apply Theorem 11 (second part) and find that, for each positive integer $n$, there is a constant $s_{n}<0$ such that for each $s<s_{n}$ equation (46) has at least $2 n$ harmonic solutions (whatever $a$ may be). We can be even more precise: for $k_{0} \in \mathbb{Z}_{0}^{+}$such that $\left(\pi k_{0} / T\right)^{2}>\lambda$, we have that the solutions we find oscillate, respectively $2 k_{0}, 2\left(k_{0}+1\right), \ldots, 2\left(k_{0}+n-1\right)$ times, around a point $c_{s}$ in a right neighborhood of $a$, such that $g\left(c_{s}\right)=s$. On the other hand, if we apply Theorem 12, we know that for each pair of positive integers, $m$ and $n$, we can find a constant $s_{m, n}<0$ such that equation (46) has at least $2 n$ periodic solutions of minimal period $m T$ for all $s<s_{m, n}$. These solutions oscillate around $c_{s}$ respectively $2 j_{1}, 2 j_{2}, \ldots, 2 j_{n}$ times in the interval [0, $m T$ [, where $j_{1}<j_{2}<\ldots<j_{n}$ is a finite sequence of integers which are prime with $m$ and with $\left(\pi j_{1} / m T\right)^{2}>\lambda$.

Example 3. As a third example, we reconsider a problem previously analysed in [18] about the dynamics of a charged particle moving on a line where one has placed one or more fixed charges at fixed positions. In [18] the case of repulsive forces was discussed. Here we examine also the situation in which some of the forces are of attractive type and other are of repulsive type.

Following [18] we suppose at first that an electric charge $Q$ is fixed at a point $d \in \mathbb{R}$. Let $q$ be another charge of the same sign of $Q$ which is free to move on one side with respect to $d$ and denote by $x(t)$ the position of $q$ at the time $t$. Assuming an external forcing of the form $p(t)=s+e(t)$ with $e(\cdot)$ as in the previous examples, we are led to consider the equation

$$
x^{\prime \prime}-\kappa_{0} \frac{x-d}{|x-d|^{3}}=s+e(t),
$$


with $\kappa_{0}>0$ a fixed constant (see [18]). Equation (48) presents a singularity of repulsive type at the point $x=d$. It follows from a theorem of Lazer and Solimini in [28], that (48) has a $T$-periodic solution if and only if $e_{s}:=s+T^{-1} \int_{0}^{T} e(t) d t \neq 0$. Moreover, such a solution lies in $] d,+\infty)$ or $(-\infty, d[$, according to the fact that $e_{s}<0$ or $e_{s}>0$ (for related results see also [22], [32] and the references therein). As a consequence of [18], equation (48) has at least one $m$-th order subharmonic solution for each $m$ sufficiently large. We see now what happens when $|s|$ becomes large according to our results. If the charge $q$ moves at the left of $d$, then we can apply Theorem 7 for $s>0$ with $b=d$ and $\alpha=0<\gamma=+\infty$, while, if we are interested in the motion of the free charge at the right of $d$, we apply Theorem 11 for $s<0$ with $a=d$ and $\alpha=0<\gamma=+\infty$. Thus, in any case, we find that for each positive integer $n$, there is a constant $s_{n}>0$ such that equation (48) has at least $2 n$ harmonic solutions for all $s$ with $|s|>s_{n}$. These solutions lie in the interval $] d,+\infty)$ if $s<-s_{n}$, while they are in $\left(-\infty, d\left[\right.\right.$ if $s>s_{n}$. Moreover, they oscillate around a point in a right/left neighborhood of $d$, respectively $2,4, \ldots, 2 n$ times in the interval [0,T[. Furthermore, we can apply also Theorems 10 and 12 and obtain that for each pair of positive integers, $m$ and $n$, there is a constant $s_{m, n}>0$ such that equation (48) has at least $2 n$ periodic solutions of minimal period $m T$ for each $s$ with $|s|>s_{m, n}$. These solutions lie in the interval $\left.] d,+\infty\right)$ if $s<-s_{m, n}$, while they are in $\left(-\infty, d\left[\right.\right.$ if $s>s_{m, n}$ and oscillate around a point in a right/left neighborhood of $d$, respectively $2 j_{1}, 2 j_{2}, \ldots, 2 j_{n}$ times in the interval [0, $m T$ [, where $j_{1}<j_{2}<\ldots<j_{n}$ is a finite sequence of integers which are prime with $m$.

In [18, Example 4.2], the case of a particle moving between two charges of the same sign was considered too. In that case, we cannot tell anything new with respect to [18, Proposition 4.2]. Then we examine the case in which the charges have opposite sign. Suppose that two electric charges $Q_{1}$ and $Q_{2}$ of opposite sign are placed at the points $a$ and $b$, respectively, with $-\infty<a<b<+\infty$. Let $q$ be another charge which is free to move between $a$ and $b$ and denote by $x(t)$ its position at the time $t$. Assuming, as above, an external forcing of the form $p(t)=s+e(t)$ with $e(\cdot)$ continuous and periodic of minimal period $T>0$, we are led to consider the equation

$$
x^{\prime \prime}-\frac{\kappa_{1}}{|x-a|^{2}}+\frac{\kappa_{2}}{|x-b|^{2}}=s+e(t)
$$

with $\kappa_{1}$ and $\kappa_{2}$ nonzero constants having the same sign of $Q_{1} q$ and $Q_{2} q$, respectively. Now, if $\kappa_{1}<0<\kappa_{2}$ we are in the setting of Corollary 11, whence if $\kappa_{1}>0>\kappa_{2}$, a corresponding dual result from Section 8 can be applied. In the former case, we conclude precisely as for equation (45) above and thus we have that for any positive integer $n$, there is a constant $s_{n}>0$ such that equation (49) has at least $2 n+1$ harmonic solutions for all $s>s_{n}$. One of these solutions lies near the attractive singularity at $a$, while all the other ones oscillate around a point in a neighborhood of the repulsive singularity at $b$. Subharmonic solutions of any order appear by virtue of Corollary 12, as well. The structure of the set of subharmonic solutions is similar to that described previously for equation (45). In the latter case, when a repulsive force acts from $a$ and an attractive one from $b$, we obtain an analogous result for $s$ negative with $|s|$ large. 


\section{REFERENCES}

1. A. Ambrosetti and G. Prodi, On the inversion of some differentiable mappings with singularities between Banach spaces, Ann. Mat. Pura Appl. 93 (1972), 231-247. MR 47:9377

2. M. Berger and E. Podolak, On the solutions of a nonlinear Dirichlet problem, Indiana Univ. Math. J. 24 (1975), 837-846. MR 51:13447

3. A. Castro and R. Shivaji, Multiple solutions for a Dirichlet problem with jumping nonlinearities, II, J. Math. Anal. Appl. 133 (1988), 509-528. MR 89e:34031

4. R. Conti, Soluzioni periodiche dell'equazione di Liénard generalizzata. Esistenza ed unicità, Boll. Un. Mat. Ital. 3 (1952), 111-118. MR 14:558a

5. D.G. Costa, D.G. De Figueiredo and P.N. Srikanth, The exact number of solutions for a class of ordinary differential equations through Morse index computation, J. Differential Equations 96 (1992), 195-199. MR 93c:34050

6. E.N. Dancer, Boundary value problems for weakly nonlinear ordinary differential equations, Bull. Austral. Math. Soc. 15 (1976), 321-328. MR 55:3389

7. C. De Coster, La méthode des sur et sous solutions dans l'étude de problèmes aux limites, $\mathrm{Ph}$. D. Thesis, Université Catholique de Louvain, 1994.

8. M. del Pino, R. F. Manásevich and A. Murua, On the number of $2 \pi$ periodic solutions for $u^{\prime \prime}+g(u)=s(1+h(t))$ using the Poincaré-Birkhoff theorem, J. Differential Equations 95 (1992), 240-258. MR 93e:34062

9. M. del Pino, R. Manásevich and A. Montero, T-periodic solutions for some second order differential equations with singularities, Proc. Roy. Soc. of Edinburgh 120 A (1992), 231-243. MR 93c:34091

10. W.Y. Ding, A generalization of the Poincaré-Birkhoff theorem, Proc. Amer. Math. Soc. 88 (1983), 341-346. MR 84f:54053

11. T. Ding and F. Zanolin, Periodic solutions of Duffing's equations with superquadratic potential, J. Differential Equations 97 (1992), 328-378. MR 93e:34059

12. T. Ding and F. Zanolin, Periodic solutions and subharmonic solutions for a class of planar systems of Lotka-Volterra type, Proc. of the 1st World Congress of Nonlinear Analysts (V. Lakshmikantham, ed.), Tampa 1992 (to appear).

13. T. Ding, R. Iannacci and F. Zanolin, Existence and multiplicity results for periodic solutions of semilinear Duffing equations, J. Differential Equations 105 (1993), 364-409. MR 94g:34060

14. C. Fabry and P. Habets, Periodic solutions of second order differential equations with superlinear asymmetric nonlinearities, Arch. Math. 60 (1993), 266-276. MR 93j:34055

15. C. Fabry, J. Mawhin and M. N. Nkashama, A multiplicity result for periodic solutions of forced nonlinear second order ordinary differential equations, Bull. London Math. Soc. 18 (1986), 173-180. MR 87e:34072

16. D.G. Figueiredo and B. Ruf, On the periodic Fučik spectrum and a superlinear Sturm Liouville equation, Proc. Roy. Soc. of Edinburgh 123 A (1993), 95-107. MR 93m:34027

17. A. Fonda and A. C. Lazer, Subharmonic solutions of conservative systems with nonconvex potentials, Proc. Amer. Math. Soc. 115 (1992), 183-190. MR 92h:34082

18. A. Fonda, R. Manásevich and F. Zanolin, Subharmonic solutions for some second-order differential equations with singularities, SIAM J. Math. Anal. 24 (1993), 1294-1311. MR 94f:34085

19. A. Fonda and M. Ramos, Large-amplitude subharmonic oscillations for scalar second-order differential equations with asymmetric nonlinearities, J. Differential Equations 109 (1994), 354-372. MR 95e:34032

20. A. Fonda, M. Ramos and M. Willem, Subharmonic solutions for second order differential equations, Topological Methods in Nonlinear Analysis 1 (1993), 49-66. MR 94c:58027

21. S. Fučik, Solvability of nonlinear equations and boundary value problems, Reidel, Dordrecht, 1980. MR 83c: 47079

22. P. Habets and L. Sanchez, Periodic solutions of some Liénard equations with singularities, Proc. Amer. Math. Soc. 109 (1990), 1035-1044. MR 90k:34049

23. D. C. Hart, A. C. Lazer and P. J. McKenna, Multiplicity of solutions of nonlinear boundary value problems, SIAM J. Math. Anal. 17 (1986), 1332-1338. MR 87k:34027

24. L. Hua, Introduction to number theory, Springer-Verlag, Berlin, 1982. MR 83f:10001

25. A.C. Lazer and P.J. McKenna, Large scale oscillatory behaviour in loaded asymmetric systems, Ann. Inst. H. Poincaré Anal. Non Linéaire, 4 (1987), 243-274. MR 88m:58028 
26. A.C. Lazer and P.J. McKenna, Existence, uniqueness, and stability of oscillations in differential equations with asymmetric nonlinearities, Trans. Amer. Math. Soc. 315 (1989), 721-739. MR 90a:34011

27. A.C. Lazer and P.J. McKenna, Large-amplitude periodic oscillations in suspension bridges: some new connections with nonlinear analysis, SIAM Review 32 (1990), 537-578. MR 92g: 73059

28. A.C. Lazer and S. Solimini, On periodic solutions of nonlinear differential equations with singularities, Proc. Amer. Math. Soc. 88 (1987), 109-114. MR 87k:34064

29. D. Lupo, S. Solimini and P. N. Srikanth, Multiplicity results for an ODE problem with even nonlinearity, Nonlinear Anal. TMA 12 (1988), 657-673. MR 89j:34026

30. H.G. Kaper and M.K. Kwong, On two conjectures concerning the multiplicity of solutions of a Dirichlet problem, SIAM J. Math. Anal. 23 (1992), 571-578. MR 93c:34055

31. W. Massey, Algebraic topology: an introduction, Springer-Verlag, Berlin, 1984.

32. J. Mawhin, Continuation principles and boundary value problems, Topological methods for ordinary differential equations (Furi and Zecca, eds.), C.I.M.E., Montecatini 1991, vol. 1537, LNM Springer-Verlag, Berlin, 1993, pp. 74-142. MR 94h:47121

33. J. Mawhin and M. Willem, Critical point theory and Hamiltonian systems, Springer-Verlag, New York, 1989. MR 90e:58016

34. R. Michalek and G. Tarantello, Subharmonic solutions with prescribed minimal period for nonautonomous Hamiltonian systems, J. Differential Equations 72 (1988), 28-55. MR 89c: 58040

35. W. Neumann, Generalizations of the Poincaré-Birkhoff fixed point theorem, Bull. Austral. Math. Soc. 17 (1977), 375-389. MR 58:28435

36. R. Ortega, Stability of a periodic problem of Ambrosetti - Prodi type, J. Differential and Integral Equations 3 (1990), 275-284. MR 90m:34104

37. M. Pei, Mather sets for twist maps and Duffing equations, preprint.

38. V. Pliss, Nonlocal problems of the theory of oscillations, Academic Press, New York, 1966. MR 33:4391

39. C. Rebelo, Ph. D. Thesis, in preparation.

40. K. Schmitt, Boundary value problems with jumping nonlinearities, Rocky Mountain J. Math. 16 (1986), 481-496. MR 87m:34016

41. J. You, Boundedness for solutions of superlinear Duffing equations via the twist theorem, Science in China (A) 35 (1992), 399-412. MR 95c:34067

42. F. Zanolin, Continuation theorems for the periodic problem via the translation operator, Rend. Sem. Mat. Univ. Torino (to appear).

43. B. Zinner, Multiplicity of solutions for a class of superlinear Sturm-Liouville problems, J. Math. Anal. Appl. 176 (1993), 282-291. MR 94j:34026

International School for Advanced Studies, via Beirut 2-4, 34013 Trieste, Italy Current address: Centro de Matemática e Aplicações Fundamentais, Av. Prof. Gama Pinto 2, 1699 Lisboa Codex, Portugal

E-mail address: carlota@ptmat.lmc.fc.ul.pt

Dipartimento di Matematica e Informatica, Università, via delle Scienze 208 (Loc. Rizzi), 33100 Udine, ItALY

E-mail address: zanolin@dimi.uniud.it 